\title{
Total Synthesis of $( \pm)$-Thebainone A by Intramolecular Nitrone Cycloaddition
}

Yuzhou Wang, André Hennig, Thomas Küttler, Christian Hahn, Anne Jäger, and Peter Metz*

Fakultät Chemie und Lebensmittelchemie, Organische Chemie I, Technische Universität Dresden, Bergstrasse 66, 01069 Dresden, Germany

Supporting Information 


\section{Table of contents}

1. General information $\quad$ S3

2. Preparation of acetal $\mathbf{1 0} \quad$ S3

3. Preparation of ester $12 \quad$ S4

4. Heck cyclization to give lactone 5

5. Preparation of dihydroxy phenol 4 via intramolecular nitrone cycloaddition S6

6. Preparation of silylether $15 \quad$ S7

7. Preparation of diacetate $16 \quad$ S8

8. Preparation of tosylate $\mathbf{1 8}$ S9

9. Preparation of morphinan $20 \quad S 10$

10. Preparation of cyclohexene $22 \quad$ S11

11. Preparation of ethyl carbamate $23 \quad$ S12

12. Preparation of cyclohexenone $\mathbf{2 4}$ by allylic oxidation $\quad \mathrm{S} 13$

13. Reduction of carbamate 27 to give $N$-methyl phenol SI-5 S15

14. Preparation of dioxolane $25 \quad$ S17

15. Preparation of acetal $26 \quad$ S18

16. Preparation of $( \pm)$-thebainone A (1) S19

$\begin{array}{ll}\text { 17. References } & \text { S20 }\end{array}$

$\begin{array}{ll}\text { 18. NMR spectra } & \text { S21 }\end{array}$ 


\section{General information}

THF and $\mathrm{CH}_{2} \mathrm{Cl}_{2}$ were dried and purified by passage through a MB-SPS-800 device using molecular sieves. Et $3 \mathrm{~N}$ was freshly distilled over $\mathrm{CaH}_{2}$ and 1,4-dioxane over $\mathrm{LiAlH}_{4}$. All other commercially available reagents were used as received. Reactions were performed under argon atmosphere. Thin layer chromatography (TLC) was performed on Merck silica gel $60 \mathrm{~F}_{254} 0.2$ $\mathrm{mm}$ precoated plates. Product spots were visualized by UV light at $254 \mathrm{~nm}$ and subsequently developed using anisaldehyde solution as appropriate. Flash column chromatography was carried out using silica gel (Merck, particle size 40-63 microns). Melting points were measured on a Wagner \& Munz PolyTherm A and are uncorrected. Infrared spectra were recorded on a THERMONICOLET Avatar 360 instrument using ATR. NMR spectra were recorded on a Bruker AC $300 \mathrm{P}\left(300 \mathrm{MHz}{ }^{1} \mathrm{H}, 75 \mathrm{MHz}{ }^{13} \mathrm{C}\right)$, on a Bruker DRX $500 \mathrm{P}\left(500 \mathrm{MHz}{ }^{1} \mathrm{H}, 125 \mathrm{MHz}\right.$ $\left.{ }^{13} \mathrm{C}\right)$ or on a Bruker AC $600-\mathrm{P}\left(600 \mathrm{MHz}{ }^{1} \mathrm{H}, 151 \mathrm{MHz}{ }^{13} \mathrm{C}\right)$ spectrometer. Chemical shifts $(\delta)$ are quoted in parts per million (ppm) downfield of tetramethylsilane, using residual protoncontaining solvent as internal standard $\left(\mathrm{CDCl}_{3}\right.$ at $\left.7.26 \mathrm{ppm}\right)$. Abbreviations used in the description of resonances are: $\mathrm{s}$ (singlet), $\mathrm{d}$ (doublet), $\mathrm{t}$ (triplet), $\mathrm{q}$ (quartet), br (broad). Coupling constants $(J)$ are quoted to the nearest $0.1 \mathrm{~Hz}$. Mass spectra were recorded with an Agilent $5973 \mathrm{~N}$ detector coupled with an Agilent 6890N GC (GC-MS, $70 \mathrm{eV}$ ) or else with a Bruker Esquire-LC (direct injection as a methanolic $\mathrm{NH}_{4} \mathrm{OAc}$ solution, ESI). HRMS spectra were recorded on a Waters Xevo G2-XS QTof (ESI-TOF). Elemental analysis was performed on a Hekatech EA 3000.

\section{Preparation of acetal 10}
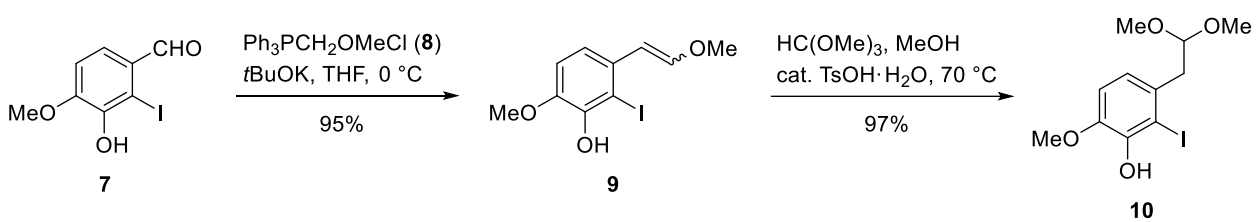

A solution of KOtBu $(4.74 \mathrm{~g}, 38.8 \mathrm{mmol})$ in THF $(60 \mathrm{~mL})$ was added by cannula to a suspension of aryl iodide $7^{1}(4.150 \mathrm{~g}, 14.93 \mathrm{mmol})$ and $8.19 \mathrm{~g}(23.9 \mathrm{mmol})$ of the phosphonium salt 8 in THF $(60 \mathrm{~mL})$ at $0{ }^{\circ} \mathrm{C}$. After about 1 equivalent of base was added, the mixture turned deep red. The mixture was stirred for $3 \mathrm{~h}$, diluted with water $(60 \mathrm{~mL})$, and neutralized with $2 \mathrm{~N} \mathrm{HCl}$. After separation of the organic layer, the aqueous layer was extracted with diethyl ether $(3 \times 60$ $\mathrm{mL}$ ). The combined organic layers were washed with saturated aqueous $\mathrm{NaCl}$ solution and dried over $\mathrm{MgSO}_{4}$. After removal of the solvent under vacuum, the residue was purified by flash chromatography over silica gel (pentane/Et ${ }_{2} \mathrm{O}, 3: 1$ ) to give an $\mathrm{E} / \mathrm{Z}=1: 0.6$ mixture of enol ether $9(4.353 \mathrm{~g}, 95 \%)$ as a white solid. 
To a solution of enol ether 9 (4.353 g, $14.22 \mathrm{mmol})$ obtained as described above in dry $\mathrm{MeOH}$ $(15 \mathrm{~mL})$ were added trimethyl orthoformate $(3.10 \mathrm{~mL}, 28.4 \mathrm{mmol})$ and $p$-toluenesulfonic acid monohydrate $(0.202 \mathrm{~g}, 1.07 \mathrm{mmol})$. After stirring the reaction mixture at $70{ }^{\circ} \mathrm{C}$ for $21 \mathrm{~h}$, it was cooled to $0{ }^{\circ} \mathrm{C}$, diluted with water $(50 \mathrm{~mL})$, and excess $\mathrm{NaCl}$ was added. The mixture was extracted with diethyl ether $(4 \times 30 \mathrm{~mL})$, and the combined organic layers were washed with saturated aqueous $\mathrm{NaCl}$ solution $(2 \times 20 \mathrm{~mL})$. After drying over $\mathrm{MgSO}_{4}$ and removal of the solvent under vacuum, the crude product was purified by flash chromatography over silica gel (pentane/Et $2 \mathrm{O}, 1: 1)$ to yield acetal $\mathbf{1 0}^{2}(4.669 \mathrm{~g}, 97 \%)$ as a slightly yellow solid.

10: $\mathrm{R}_{f}=0.37$ (pentane/Et $\left.2 \mathrm{O}, 1: 1\right)$. M.p. $=87-88{ }^{\circ} \mathrm{C} .{ }^{1} \mathrm{H}$ NMR $\left(300 \mathrm{MHz}, \mathrm{CDCl}_{3}\right): \delta=3.04(\mathrm{~d}$, $J=5.6 \mathrm{~Hz}, 2 \mathrm{H}) 3.28-3.40(\mathrm{~m}, 6 \mathrm{H}) 3.89(\mathrm{~s}, 3 \mathrm{H}) 4.58(\mathrm{t}, J=5.6 \mathrm{~Hz}, 1 \mathrm{H}) 6.19(\mathrm{~s}, 1 \mathrm{H}), 6.78$ $(\mathrm{d}, J=8.3 \mathrm{~Hz}, 1 \mathrm{H}) 6.84(\mathrm{~d}, J=8.3 \mathrm{~Hz}, 1 \mathrm{H}) \mathrm{ppm} .{ }^{13} \mathrm{C} \mathrm{NMR}\left(75 \mathrm{MHz}, \mathrm{CDCl}_{3}\right): \delta=43.5(\mathrm{t})$, 54.0 (q, 2 C), 56.3 (q), 88.7 (s), 104.3 (d), 110.3 (d), 122.1 (d), 132.6 (s), 144.4 (s), 145.4 (s) ppm. IR (ATR): v = 3323 (br, m), 3008 (w), 2944 (w), 2895 (m), 2831 (w), 1597 (w), 1484 (s), 1445 (m), 1330 (w), 1276 (s), 1214 (s), 1200 (m), 1112 (s), 1068 (s), 1027 (s), 999 (m), 922 (w), $838(\mathrm{~m}), 804$ (s) cm ${ }^{-1}$. GC-MS (EI): m/z (\%) = 338 (5) [M] $]^{+}, 307$ (8), 263 (8), 180 (9), 165 (5), 75 (100), 47 (7).

\section{Preparation of ester 12}

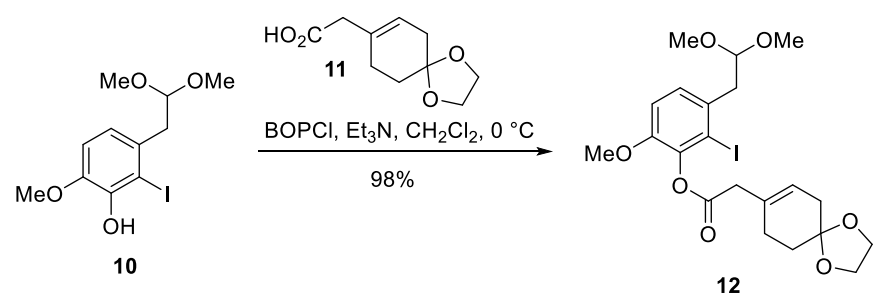

Acid $\mathbf{1 1}^{3}$ (4.25 g, $\left.21.5 \mathrm{mmol}\right)$ and $\mathrm{BOPCl}(5.71 \mathrm{~g}, 22.4 \mathrm{mmol})$ were suspended in triethylamine $(7.16 \mathrm{~mL})$ and $\mathrm{CH}_{2} \mathrm{Cl}_{2}(20 \mathrm{~mL})$ at $0^{\circ} \mathrm{C}$. After stirring the mixture for $30 \mathrm{~min}$, a solution of phenol 10 (6.60 g, $19.5 \mathrm{mmol})$ in $\mathrm{CH}_{2} \mathrm{Cl}_{2}(20 \mathrm{~mL})$ was added, and stirring was continued for 2 h at $0{ }^{\circ} \mathrm{C}$. Water $(70 \mathrm{~mL})$ was added, and the mixture was extracted with $\mathrm{CH}_{2} \mathrm{Cl}_{2}(4 \times 20 \mathrm{~mL})$. The combined organic layers were washed with saturated aqueous $\mathrm{NH}_{4} \mathrm{Cl}$ solution $(30 \mathrm{~mL})$, dried over $\mathrm{MgSO}_{4}$, and concentrated under vacuum. Flash chromatography over silica gel (pentane/Et $2 \mathrm{O}, 1: 4)$ of the residue gave ester $12(9.93 \mathrm{~g}, 98 \%)$ as a white solid.

12: $\mathrm{R}_{f}=0.36$ (pentane/Et $\left.2 \mathrm{O}, 1: 4\right)$. M.p. $=77-79{ }^{\circ} \mathrm{C} .{ }^{1} \mathrm{H}$ NMR $\left(600 \mathrm{MHz}, \mathrm{CDCl}_{3}\right): \delta=1.84(\mathrm{t}$, $J=6.6 \mathrm{~Hz}, 2 \mathrm{H}), 2.35$ (br s, $2 \mathrm{H}), 2.42-2.46$ (m, $2 \mathrm{H}), 3.04$ (d, $J=5.6 \mathrm{~Hz}, 2 \mathrm{H}), 3.33$ (br s, 2 
H), 3.34 (s, $6 \mathrm{H}), 3.79$ (s, $3 \mathrm{H}), 3.98-4.00(\mathrm{~m}, 4 \mathrm{H}), 4.53$ (t, $J=5.6 \mathrm{~Hz}, 1 \mathrm{H}), 5.65-5.69$ (m, 1 $\mathrm{H}), 6.87(\mathrm{~d}, J=8.3 \mathrm{~Hz}, 1 \mathrm{H}), 7.14(\mathrm{~d}, J=8.3 \mathrm{~Hz}, 1 \mathrm{H}) \mathrm{ppm} .{ }^{13} \mathrm{C} \mathrm{NMR}\left(151 \mathrm{MHz}, \mathrm{CDCl}_{3}\right): \delta=$ $27.6(\mathrm{t}), 31.1(\mathrm{t}), 35.8(\mathrm{t}), 42.4(\mathrm{t}), 43.7(\mathrm{t}), 54.1(\mathrm{q}), 56.1(\mathrm{q}), 64.4(\mathrm{t}), 98.8(\mathrm{~s}), 104.3(\mathrm{~d}), 107.7$ (s), 111.9 (d), 124.1 (d), 128.5 (d), 130.2 (s), 132.7 (s), 140.5 (s), 149.9 (s), 168.5 (s) ppm. IR (ATR): $v=2930(\mathrm{~m}), 2832(\mathrm{w}), 1765$ (m), 1597 (m), 1482 (s), 1439 (w), 1360 (m), 1275 (s), 1232 (m), 1192 (w), 1103 (s), 1060 (s), 1029 (s), 946 (w), 863 (m), 804 (s) cm-1 GC-MS (EI): $\mathrm{m} / \mathrm{z}(\%)=518(1)[\mathrm{M}]^{+}, 486(1), 458$ (1), 306 (15), 291 (3), 263 (7), 153 (14), 99 (5), 86 (7), 75 (100). Anal. calcd for $\mathrm{C}_{21} \mathrm{H}_{27} \mathrm{IO}_{7}$ : C, 48.66; H, 5.25; found: C, 48.85; H, 5.44.

\section{Heck cyclization to give lactone 5}

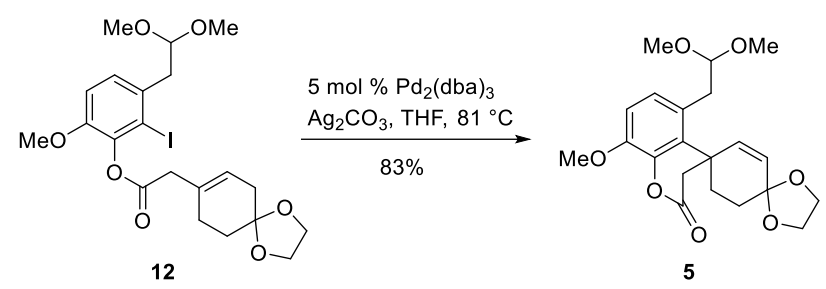

Ester 12 (1.554 g, $2.998 \mathrm{mmol}), 137.3 \mathrm{mg} \mathrm{Pd} 2(\mathrm{dba})_{3}\left(0.150 \mathrm{mmol}, 5 \mathrm{~mol} \%\right.$ ), and $\mathrm{Ag}_{2} \mathrm{CO}_{3}(2.48$ $\mathrm{g}, 8.99 \mathrm{mmol})$ were suspended in freshly dried THF $(45 \mathrm{~mL})$. The reaction flask was closed, protected from light with aluminum foil, and stirred at $81{ }^{\circ} \mathrm{C}$ oil bath temperature for $4 \mathrm{~d}$. After cooling and removal of the solvent under vacuum, the remainder was filtered through silica gel and eluted with $2 \%$ triethylamine in $\mathrm{Et}_{2} \mathrm{O}$. The crude product obtained after concentration under vacuum was purified by flash chromatography over silica gel (pentane/Et ${ }_{2} \mathrm{O}, 1: 4$ ) to afford the cyclization product $5(0.970 \mathrm{~g}, 83 \%)$ as a white solid.

5: $\mathrm{R}_{f}=0.20$ (pentane/Et $\left.2 \mathrm{O}, 1: 4\right)$. M.p. $=100-101{ }^{\circ} \mathrm{C} .{ }^{1} \mathrm{H}$ NMR $\left(600 \mathrm{MHz}, \mathrm{CDCl}_{3}\right): \delta=1.83-$ $1.88(\mathrm{~m}, 1 \mathrm{H}), 1.89-1.94(\mathrm{~m}, 1 \mathrm{H}), 1.99$ (td, $J=13.9,3.4 \mathrm{~Hz}, 1 \mathrm{H}), 2.18$ (m, $1 \mathrm{H}), 2.58$ (dd, $J=$ 15.8, $1.5 \mathrm{~Hz}, 1 \mathrm{H}), 2.84$ (d, $J=15.8 \mathrm{~Hz}, 1 \mathrm{H}), 3.03$ (dd, $J=14.7,5.6 \mathrm{~Hz}, 1 \mathrm{H}), 3.19$ (dd, $J=$ 14.7, $5.3 \mathrm{~Hz}, 1 \mathrm{H}), 3.32$ (s, $3 \mathrm{H}), 3.33$ (s, $3 \mathrm{H}), 3.86$ (s, $3 \mathrm{H}), 3.87-3.93$ (m, $1 \mathrm{H}), 3.97-4.07$ (m, $3 \mathrm{H}), 4.58(\mathrm{t}, J=5.6 \mathrm{~Hz}, 1 \mathrm{H}), 5.75(\mathrm{dd}, J=10.2,1.5 \mathrm{~Hz}, 1 \mathrm{H}), 5.96(\mathrm{dd}, J=10.2,1.9 \mathrm{~Hz}, 1 \mathrm{H})$, $6.85(\mathrm{~d}, J=8.7 \mathrm{~Hz}, 1 \mathrm{H}), 7.06(\mathrm{~d}, J=8.7 \mathrm{~Hz}, 1 \mathrm{H}) \mathrm{ppm} .{ }^{13} \mathrm{C} \mathrm{NMR}\left(151 \mathrm{MHz}, \mathrm{CDCl}_{3}\right): \delta=29.4$ $(\mathrm{t}), 30.1(\mathrm{t}), 35.3(\mathrm{t}), 38.5(\mathrm{t}), 39.5(\mathrm{~s}), 53.0(\mathrm{q}), 53.5(\mathrm{q}), 56.0(\mathrm{q}), 64.3(\mathrm{t}), 64.8(\mathrm{q}), 104.0(\mathrm{~s})$, 105.0 (d), 111.2 (d), 127.0 (s), 127.6 (d), 128.0 (d), 129.3 (s), 137.2 (d), 141.0 (s), 146.4 (s), 166.2 (s) ppm. IR (ATR): v = 2938 (m), 2896 (w), 2835 (w), 1761 (s), 1611 (w), 1578 (w), 1489 (m), 1442 (w), 1426 (w), 1285 (m), 1258 (m), 1222 (s), 1193 (s), 1118 (s), 1085 (s), 1046 (s), 981 (s), 948 (m), 888 (m), 802 (m), 652 (w) cm ${ }^{-1}$. GC-MS (EI): m/z (\%) = 390 (1) [M] $]^{+}$, 
359 (1), 327 (1), 241 (1), 213 (1), 197 (1), 177 (2), 156 (2), 141 (3), 128 (7), 115 (4), 99 (3), 86

(7), 75 (100), 47 (5). Anal. calcd for $\mathrm{C}_{21} \mathrm{H}_{26} \mathrm{O}_{7}$ : C, 64.60; H, 6.71; found: C, 64.64; H, 6.62.

\section{Preparation of dihydroxy phenol 4 via intramolecular nitrone cycloaddition}
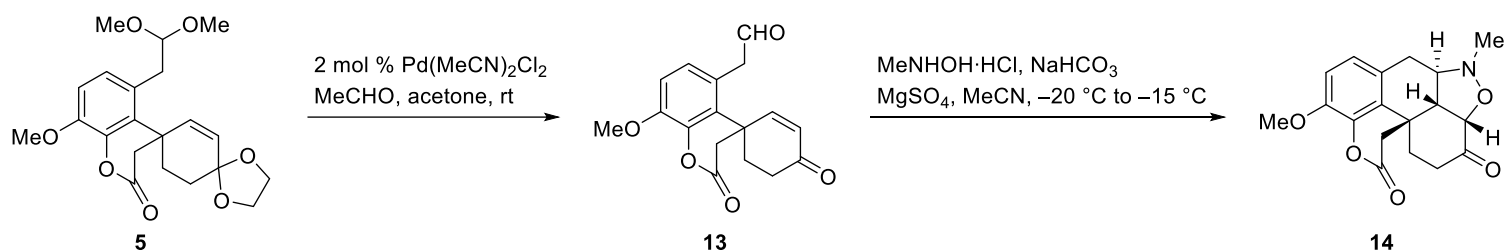

14

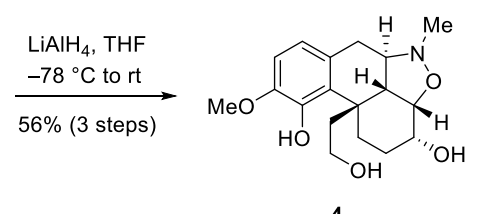

Lactone 5 (1.171 g, $2.999 \mathrm{mmol})$ was added to a solution of $\mathrm{PdCl}_{2}\left(\mathrm{MeCN}_{2}(15.56 \mathrm{mg}, 0.060\right.$ $\mathrm{mmol})$ and acetaldehyde $(3.39 \mathrm{~mL}, 60 \mathrm{mmol})$ in acetone $(90 \mathrm{~mL})$. After stirring for $6 \mathrm{~h}$ at room temperature, the reaction mixture was diluted with water $(75 \mathrm{~mL})$ and diethyl ether $(30 \mathrm{~mL})$, excess $\mathrm{NaCl}$ was added, and the mixture was extracted with diethyl ether $(4 \times 25 \mathrm{~mL})$. The combined organic layers were washed with saturated aqueous $\mathrm{NaCl}$ solution $(2 \times 20 \mathrm{~mL})$ and dried over $\mathrm{MgSO}_{4}$. After removal of the solvent under vacuum, the crude keto aldehyde $\mathbf{1 3}$ $(0.977 \mathrm{~g})$ was obtained as a yellowish foam. A small portion $(9 \mathrm{mg})$ was used for analytical purposes, and the rest was used directly for the following step.

To a suspension of $\mathrm{N}$-methylhydroxylamine hydrochloride $(275.5 \mathrm{mg}, 3.300 \mathrm{mmol})$ in acetonitrile $(60 \mathrm{~mL})$ were added $\mathrm{NaHCO}_{3}(756.0 \mathrm{mg}, 9.00 \mathrm{mmol})$ and $\mathrm{MgSO}_{4}(1.08 \mathrm{~g}, 9.00$ mmol). After stirring for $10 \mathrm{~min}$ at room temperature, the mixture was cooled to $-20{ }^{\circ} \mathrm{C}$ and treated with a solution of the crude keto aldehyde $\mathbf{1 3}(0.968 \mathrm{~g})$ in acetonitrile $(20 \mathrm{~mL})$. Stirring was continued for $9 \mathrm{~h}$ at $-15{ }^{\circ} \mathrm{C}$, water $(50 \mathrm{~mL})$ and $\mathrm{CH}_{2} \mathrm{Cl}_{2}(20 \mathrm{~mL})$ were added, and the aqueous phase was extracted with $\mathrm{CH}_{2} \mathrm{Cl}_{2}(3 \times 30 \mathrm{~mL})$. The combined organic layers were washed with saturated aqueous $\mathrm{NaCl}(2 \times 20 \mathrm{~mL})$, dried over $\mathrm{MgSO}_{4}$, and the solvent was removed under high vacuum to give the crude isoxazolidine 14 (1.048 g), which was used without further purification for the following step.

A solution of the crude isoxazolidine $14(1.048 \mathrm{~g})$ in dry THF $(50 \mathrm{~mL})$ was cooled to $-78{ }^{\circ} \mathrm{C}$ and treated with lithium aluminum hydride $(0.570 \mathrm{~g}, 15.0 \mathrm{mmol})$. Stirring was continued for 1 $\mathrm{h}$ at $-78^{\circ} \mathrm{C}$ and during warming to room temperature over further $2 \mathrm{~h}$. The mixture was then cooled to $0{ }^{\circ} \mathrm{C}$, and $\mathrm{Na}_{2} \mathrm{SO}_{4}$ decahydrate (Glauber's salt) (3.864 g) was added in order to destroy excess hydride within $30 \mathrm{~min}$. After the first gas evolution had ceased, the cooling bath was 
removed, ammonium chloride (1.60 g) was added, and the reaction mixture was allowed to warm to room temperature. Subsequently, the mixture was filtered over silica gel and eluted with THF/methanol (4:1). The solvent was removed under vacuum, and the residue was purified by flash chromatography over silica gel (EtOAc/MeOH, 9:1) to yield the dihydroxy phenol 4 $(0.562 \mathrm{~g}, 56 \%$ over 3 steps from 5) as a slightly yellow solid.

4: $\mathrm{R}_{f}=0.19(\mathrm{EtOAc} / \mathrm{MeOH}, 9: 1) .{ }^{1} \mathrm{H} \mathrm{NMR}\left(600 \mathrm{MHz}, \mathrm{CDCl}_{3}\right): \delta=2.49-2.58(\mathrm{~m}, 2 \mathrm{H}) 2.64$ $(\mathrm{dd}, J=11.5,8.1 \mathrm{~Hz}, 1 \mathrm{H}), 2.68-2.75(\mathrm{~m}, 1 \mathrm{H}), 2.80(\mathrm{dd}, J=14.3,4.1 \mathrm{~Hz}, 1 \mathrm{H}), 2.86(\mathrm{~s}, 3 \mathrm{H})$, 2.86-2.91 (m, $1 \mathrm{H}), 3.35-3.48$ (m, $2 \mathrm{H}), 3.85$ (m, $1 \mathrm{H}), 3.87$ (s, $3 \mathrm{H}), 4.31$ (dd, J = 7.3, 5.1 Hz, $1 \mathrm{H}), 6.04(\mathrm{~s}, 1 \mathrm{H}), 6.61(\mathrm{~d}, J=8.3 \mathrm{~Hz}, 1 \mathrm{H}), 6.71(\mathrm{~d}, J=8.3 \mathrm{~Hz}, 1 \mathrm{H}) \mathrm{ppm} .{ }^{13} \mathrm{C}$ NMR $(151$ $\left.\mathrm{MHz}, \mathrm{CDCl}_{3}\right): \delta=24.5(\mathrm{t}), 25.2(\mathrm{t}), 35.0(\mathrm{t}), 38.7(\mathrm{t}), 40.2(\mathrm{~s}), 44.0(\mathrm{q}) 51.6(\mathrm{~d}), 56.2(\mathrm{q}), 60.6$ (t), 63.4 (d), 64.0 (d), 75.5 (d), 109.0 (d), 120.8 (d), 127.7 (s), 127.8 (s), 144.4 (s), 145.0 (s) ppm. IR (ATR): v = 3397 (br, w), 3065 (br, w), 2963 (w), 2926 (w), 2888 (w), 2829 (w), 1477 (m), 1434 (m), 1397 (w), 1365 (w), 1340 (w), 1276 (s), 1231 (s), 1062 (s), 1036 (s), 1004 (s), $976(\mathrm{~m}), 890(\mathrm{w}), 800(\mathrm{~s}) \mathrm{cm}^{-1}$. ESI-MS: m/z = $336.1[\mathrm{M}+\mathrm{H}]^{+}$. HRMS (ESI): m/z calcd for $\mathrm{C}_{18} \mathrm{H}_{26} \mathrm{NO}_{5}[\mathrm{M}+\mathrm{H}]^{+}:$336.1805; found $336.1809, \mathrm{~m} / \mathrm{z}$ calcd for $\mathrm{C}_{18} \mathrm{H}_{25} \mathrm{NNaO}_{5}[\mathrm{M}+\mathrm{Na}]^{+}$: 358.1625 ; found 358.1628 .

\section{Preparation of silylether 15}

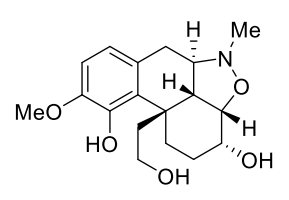

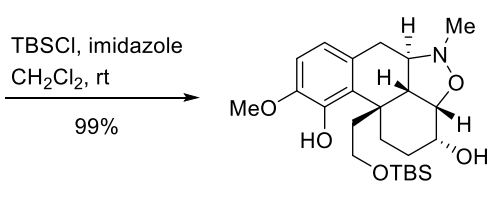

15

TBSCl (0.207 g, $1.37 \mathrm{mmol})$ was added to a suspension of dihydroxy phenol 4 (0.418 g, 1.25 mmol $)$ and imidazole $(0.212 \mathrm{~g}, 3.11 \mathrm{mmol})$ in $\mathrm{CH}_{2} \mathrm{Cl}_{2}(13 \mathrm{~mL})$. After stirring the mixture at room temperature for $4 \mathrm{~h}$, water $(3 \mathrm{~mL})$ was added. The aqueous layer was extracted with $\mathrm{CH}_{2} \mathrm{Cl}_{2}(3 \times 5 \mathrm{~mL})$, and the combined organic layers were washed with saturated aqueous $\mathrm{NaCl}$ solution and dried over $\mathrm{MgSO}_{4}$. The crude product obtained after removal of the solvent under vacuum was purified by flash chromatography over silica gel $\left(\mathrm{Et}_{2} \mathrm{O}\right)$ to give the silyl ether $\mathbf{1 5}$ $(0.556 \mathrm{~g}, 99 \%)$ as a white foam.

15: $\mathrm{R}_{f}=0.22\left(\mathrm{Et}_{2} \mathrm{O}\right) . \mathrm{M} . \mathrm{p} .=55-58{ }^{\circ} \mathrm{C} .{ }^{1} \mathrm{H}$ NMR $\left(600 \mathrm{MHz}, \mathrm{CDCl}_{3}\right): \delta=-0.08(\mathrm{~s}, 3 \mathrm{H}),-0.07$ (s, $3 \mathrm{H}), 0.81$ (s, $9 \mathrm{H}), 1.73-1.83$ (m, $2 \mathrm{H}), 1.86-1.94(\mathrm{~m}, 2 \mathrm{H}), 2.30-2.37$ (m, $1 \mathrm{H}), 2.50-2.57$ (m, 2 H), 2.61-2.70 (m, 2 H), 2.78 (dd, $J=14.3,3.8$ Hz, 1 H), 2.82-2.89 (m, 1 H), 2.86 (s, 3 
H), 3.29-3.35 (m, $1 \mathrm{H}), 3.36-3.42(\mathrm{~m}, 1 \mathrm{H}), 3.85-3.89(\mathrm{~m}, 1 \mathrm{H}), 3.87$ (s, $3 \mathrm{H}), 4.31$ (dd, $J=$ 7.2, $5.3 \mathrm{~Hz}, 1 \mathrm{H}), 5.98(\mathrm{~s}, 1 \mathrm{H}), 6.59(\mathrm{~d}, J=8.3 \mathrm{~Hz}, 1 \mathrm{H}), 6.71(\mathrm{~d}, J=8.3 \mathrm{~Hz}, 1 \mathrm{H}) \mathrm{ppm} .{ }^{13} \mathrm{C}$ NMR (151 MHz, $\left.\mathrm{CDCl}_{3}\right): \delta=-5.3(\mathrm{q}), 18.3(\mathrm{~s}), 24.5(\mathrm{t}), 25.2(\mathrm{t}), 26.0(\mathrm{q}), 35.0(\mathrm{t}), 38.7(\mathrm{t})$, 40.1 (s), 44.1 (q), 51.6 (d), 56.2 (q), 61.0 (t), 63.4 (d), 64.2 (d), 75.5 (d), 108.9 (d), 120.6 (d), 127.8 (s), 128.0 (s), 144.5 (s), 145.0 (s) ppm. IR (ATR): v = 3522 (w), 2952 (w), 2926 (w), 2928 (m), 2882 (w), 2855 (w), 1519 (w), 1480 (m), 1441 (w), 1276 (s), 1254 (s), 1083 (s), 1060 (s), $1004(\mathrm{~m}), 834$ (s), 775 (s) $\mathrm{cm}^{-1}$. ESI-MS: m/z = $450.4[\mathrm{M}+\mathrm{H}]^{+}$. HRMS (ESI): m/z calcd for $\mathrm{C}_{24} \mathrm{H}_{40} \mathrm{NO}_{5} \mathrm{Si}[\mathrm{M}+\mathrm{H}]^{+}:$450.2670; found 450.2676.

\section{Preparation of diacetate 16}

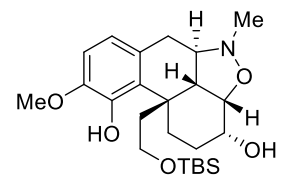

15

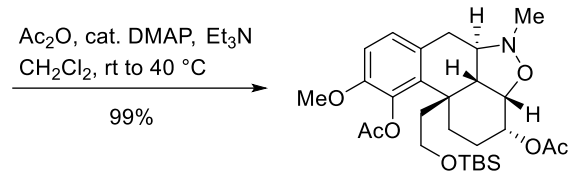

16

To a solution of silyl ether $15(0.487 \mathrm{~g}, 1.08 \mathrm{mmol})$, DMAP (0.675 g, $0.553 \mathrm{mmol})$, and triethylamine $(1.12 \mathrm{~g}, 11.1 \mathrm{mmol})$ in $\mathrm{CH}_{2} \mathrm{Cl}_{2}(8 \mathrm{~mL})$ was added acetic anhydride $(0.451 \mathrm{~g}, 4.42$ mmol). After stirring for $23 \mathrm{~h}$ at room temperature and $1 \mathrm{~h}$ at $40{ }^{\circ} \mathrm{C}$, the reaction mixture was cooled to $0{ }^{\circ} \mathrm{C}$ and treated with saturated aqueous $\mathrm{NH}_{4} \mathrm{Cl}$ solution $(5 \mathrm{~mL})$. The aqueous layer was extracted with diethyl ether $(3 \times 5 \mathrm{~mL})$, and the combined organic layers were washed with saturated aqueous $\mathrm{NaCl}$ solution $(2 \times 6 \mathrm{~mL})$ and dried over $\mathrm{MgSO}_{4}$. Removal of the solvent and flash chromatography over silica gel $\left(\mathrm{Et}_{2} \mathrm{O}\right)$ yielded diacetate $16(0.573 \mathrm{~g}, 99 \%)$ as a white foam.

16: $\mathrm{R}_{f}=0.31\left(\mathrm{Et}_{2} \mathrm{O}\right) . \mathrm{M} . \mathrm{p} .=67-69{ }^{\circ} \mathrm{C} .{ }^{1} \mathrm{H}$ NMR $\left(500 \mathrm{MHz}, \mathrm{d}_{6}\right.$-acetone $): \delta=-0.08(\mathrm{~s}, 3 \mathrm{H})$, -0.07 (s, 3 H), 0.79-0.83 (m, 9 H), 1.62 (m, 1 H), 1.70-1.90 (m, 3 H), 1.98-2.11 (m, 1 H), 2.07 (s, 3 H), 2.23-2.43 (m, 1 H), 2.32 (s, 3 H), 2.60 (t, $J=12.3$ Hz, 1 H), 2.67-2.98 (m, 3 H), 2.82 (s, 3 H), 3.30-3.43 (m, 2 H), 3.79 (s, 3 H), 4.32-4.39 (m, 1 H), 5.09 (br s, 1 H), 6.85 (d, J = 8.5 $\mathrm{Hz}, 1 \mathrm{H}), 6.99(\mathrm{~d}, J=8.5 \mathrm{~Hz}, 1 \mathrm{H}) \mathrm{ppm} .{ }^{13} \mathrm{C} \mathrm{NMR}\left(125 \mathrm{MHz}, \mathrm{CDCl}_{3}\right): \delta=-5.43$ (q), -5.39 (q), $18.2(\mathrm{~s}), 21.2(\mathrm{q}), 21.8(\mathrm{q}), 23.3(\mathrm{t}), 25.9(\mathrm{q}), 28.1(\mathrm{t}), 35.2(\mathrm{t}), 40.0(\mathrm{t}), 40.4(\mathrm{~s}), 44.1(\mathrm{q}), 51.5$ (d), 56.2 (q), 60.5 (t), 62.8 (d), 67.0 (d), 73.1 (d), 111.2 (d), 127.9 (s), 127.9 (d), 134.5 (s), 139.4 (s), 149.9 (s), 169.0 (s), 169.8 (s) ppm. IR (ATR): v = 2951 (w), 2927 (w), 2878 (w), 2849 (w), 1770 (m), 1739 (s), 1482 (m), 1473 (m), 1458 (m), 1365 (m), 1290 (m), 1239 (s), 1205 (s), 1182

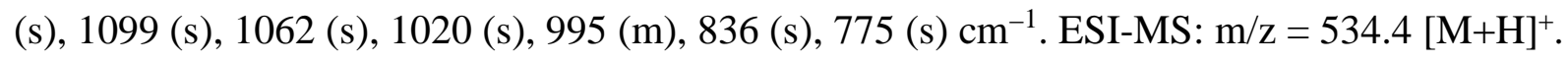
HRMS (ESI): $\mathrm{m} / \mathrm{z}$ calcd for $\mathrm{C}_{28} \mathrm{H}_{44} \mathrm{NO}_{7} \mathrm{Si}[\mathrm{M}+\mathrm{H}]^{+}$: 534.2882; found 534.2894. 


\section{Preparation of tosylate 18}

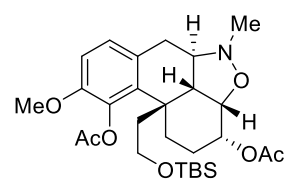

16

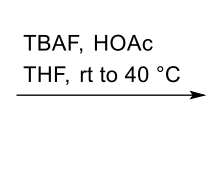

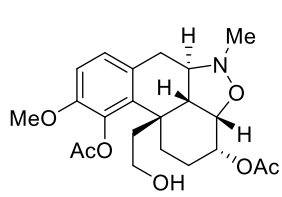

17

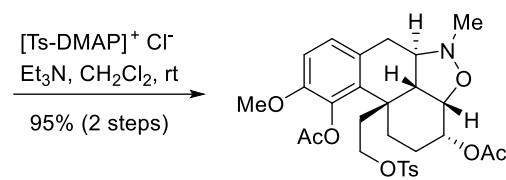

18

To a solution of diacetate $16(0.572 \mathrm{~g}, 1.07 \mathrm{mmol})$ in $\mathrm{CH}_{2} \mathrm{Cl}_{2}(10 \mathrm{~mL})$ was added dropwise at room temperature a mixture of TBAF $(1.61 \mathrm{~mL}, 1 \mathrm{M}$ in THF, $1.61 \mathrm{mmol})$ and acetic acid $(0.193$ $\mathrm{g}, 3.22 \mathrm{mmol}$ ). After stirring the mixture for $20 \mathrm{~h}$ at room temperature and for $1 \mathrm{~h}$ at $40{ }^{\circ} \mathrm{C}$, it was cooled to $0{ }^{\circ} \mathrm{C}$ and treated with saturated aqueous $\mathrm{NH}_{4} \mathrm{Cl}$ solution $(6 \mathrm{~mL})$. The aqueous layer was extracted with diethyl ether $(3 \times 6 \mathrm{~mL})$, and the combined organic layers were washed with saturated aqueous $\mathrm{NaCl}$ solution $(6 \mathrm{~mL})$ and dried over $\mathrm{MgSO}_{4}$. Removal of the solvent under vacuum and flash chromatography over silica gel (EtOAc/MeOH, 20:1 to 10:1) afforded the partially purified alcohol $17(0.467 \mathrm{~g})$ as a white foam.

17: $\mathrm{R}_{f}=0.19\left(\right.$ EtOAc/MeOH, 20:1). ESI-MS: m/z = $420.4[\mathrm{M}+\mathrm{H}]^{+}$. HRMS (ESI): m/z calcd for $\mathrm{C}_{22} \mathrm{H}_{30} \mathrm{NO}_{7}[\mathrm{M}+\mathrm{H}]^{+}$: 420.2017; found 420.2024.

To a solution of the partially purified alcohol $17(0.467 \mathrm{~g})$ obtained as described above and triethylamine $(0.216 \mathrm{~g}, 2.14 \mathrm{mmol})$ in $\mathrm{CH}_{2} \mathrm{Cl}_{2}(7.5 \mathrm{~mL})$ cooled to $0{ }^{\circ} \mathrm{C}$ was added the tosylation agent ${ }^{4}$ [Ts-DMAP $]^{+} \mathrm{Cl}^{-}(0.797 \mathrm{~g}, 1.61 \mathrm{mmol})$. After stirring for $3 \mathrm{~h}$ at room temperature, the reaction mixture was cooled to $0{ }^{\circ} \mathrm{C}$ and treated with saturated aqueous $\mathrm{NH}_{4} \mathrm{Cl}$ solution $(10$ $\mathrm{mL})$. The aqueous layer was extracted with diethyl ether $(4 \times 15 \mathrm{~mL})$, and the combined organic layers were washed with water $(10 \mathrm{~mL})$, saturated aqueous $\mathrm{NaCl}$ solution $(10 \mathrm{~mL})$, and dried over $\mathrm{MgSO}_{4}$. Removal of the solvent under vacuum and flash chromatography over silica gel (EtOAc) yielded tosylate $\mathbf{1 8}(0.583 \mathrm{~g}, 95 \%$ from 16 over 2 steps $)$ as a white foam.

18: $\mathrm{R}_{f}=0.28$ (EtOAc). M.p. $=90-91{ }^{\circ} \mathrm{C} .{ }^{1} \mathrm{H} \mathrm{NMR}\left(600 \mathrm{MHz}, \mathrm{CDCl}_{3}\right): \delta=1.53-1.68(\mathrm{~m}, 2 \mathrm{H})$, 1.78-1.89 (m, 2 H), 2.06 (s, 3 H), 2.10 (br s, 1 H), 2.30 (s, 3 H), 2.43 (s, 3 H), 2.47-2.61 (m, 3 H), 2.80 (s, 3 H), 2.81-2.94 (m, 2 H), 3.71-3.77 (m, 1 H), 3.79 (s, 3 H), 3.85 (br s, $1 \mathrm{H}), 4.23$ (br s, $1 \mathrm{H}), 5.06$ (br s, $1 \mathrm{H}), 6.84(\mathrm{~d}, J=8.1 \mathrm{~Hz}, 1 \mathrm{H}), 6.95(\mathrm{~d}, J=8.1 \mathrm{~Hz}, 1 \mathrm{H}), 7.29$ (d, $J=7.7$ $\mathrm{Hz}, 2 \mathrm{H}), 7.67$ (d, $J=7.7 \mathrm{~Hz}, 2 \mathrm{H}$ ) ppm. $\left.{ }^{13} \mathrm{C} \mathrm{NMR} \mathrm{(151} \mathrm{MHz,} \mathrm{CDCl}_{3}\right): \delta=21.1$ (q), 21.6 (q), $21.8(\mathrm{q}), 23.2(\mathrm{t}), 28.0(\mathrm{t}), 35.0(\mathrm{t}), 36.5(\mathrm{t}), 40.1(\mathrm{~s}), 44.0(\mathrm{q}), 51.4(\mathrm{~d}), 56.2(\mathrm{q}), 62.5(\mathrm{~d}), 66.6$ (d), 67.9 (t), 72.8 (d), 111.6 (d), 127.7 (s), 127.8 (d), 128.2 (d), 129.8 (d), 133.0 (s), 139.3 (s), 
144.7 (s), 150.0 (s), 168.7 (s), 169.7 (s) ppm. IR (ATR): v = 2941 (w), 2876 (w), 1766 (w), 1732 (m), 1482 (w), 1436 (w), 1361 (m), 1287 (m), 1243 (m), 1173 (s), 1096 (w), 1065 (m), $1018(\mathrm{~s}), 958(\mathrm{~s}), 878(\mathrm{~m}), 808(\mathrm{~s}), 763(\mathrm{~m}), 665(\mathrm{~s}) \mathrm{cm}^{-1}$. ESI-MS: m/z = $574.4[\mathrm{M}+\mathrm{H}]^{+}$. HRMS (ESI): $\mathrm{m} / \mathrm{z}$ calcd for $\mathrm{C}_{29} \mathrm{H}_{36} \mathrm{NO}_{9} \mathrm{~S}[\mathrm{M}+\mathrm{H}]^{+}: 574.2105$; found 574.2110.

\section{Preparation of morphinan 20}

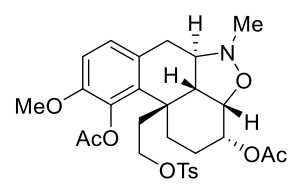

18

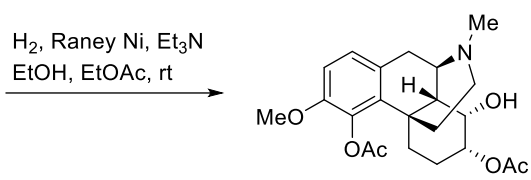

19

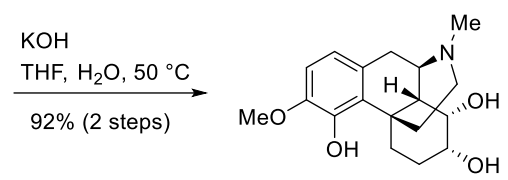

20

To a solution of tosylate $\mathbf{1 8}(1.223 \mathrm{~g}, 2.132 \mathrm{mmol})$ in EtOH/EtOAc $(3: 1,60 \mathrm{~mL})$ was added dry triethylamine $(0.59 \mathrm{~mL}, 4.2 \mathrm{mmol})$ followed by freshly prepared Raney nickel $(1.70 \mathrm{~g})$ suspended in EtOH $(20 \mathrm{~mL})$. A balloon filled with hydrogen was attached to the reaction flask, the reaction mixture was briefly flushed with hydrogen, and then the flask was closed. After stirring at room temperature for $24 \mathrm{~h}$, the mixture was flushed with nitrogen for a few minutes, and Raney nickel was removed by filtration and washed with ethanol (caution: do not allow to dry - risk of fire due to residual hydrogen on the catalyst). The combined filtrates were concentrated under vacuum, and flash chromatography of the residue $\left(\mathrm{CHCl}_{3} / \mathrm{MeOH}, 4: 1\right)$ gave the product 19 as a white foamy solid in the form of the tosyl salt. In order to remove the $p$ toluenesulfonic acid, the product was dissolved in $\mathrm{CH}_{2} \mathrm{Cl}_{2}(30 \mathrm{~mL})$, saturated aqueous $\mathrm{NaHCO}_{3}$ solution $(15 \mathrm{~mL})$ was added, and the mixture was stirred at $0{ }^{\circ} \mathrm{C}$ for $2 \mathrm{~h}$. The organic layer was washed with water, saturated aqueous $\mathrm{NaCl}$ solution, and dried over $\mathrm{MgSO}_{4}$. After removal of the solvent under vacuum, the free amine $19(790.0 \mathrm{mg})$ was obtained, which was used for the following step without further purification.

A solution of the free amine $19(790.0 \mathrm{mg})$ obtained as described above in THF (20 mL) was treated with water $(10 \mathrm{~mL})$ and $\mathrm{KOH}(0.384 \mathrm{~g}, 6.85 \mathrm{mmol})$. The reaction mixture was stirred for $3 \mathrm{~h}$ at $50{ }^{\circ} \mathrm{C}$ oil bath temperature and then overnight at room temperature. After cooling to $0{ }^{\circ} \mathrm{C}$, the mixture was neutralized with $2 \mathrm{~N} \mathrm{HCl}$ and saturated with excess $\mathrm{NaCl}$. The aqueous layer was extracted with $\mathrm{CHCl}_{3} /$ isopropanol $(4: 1,15 \times 10 \mathrm{~mL})$, and the combined organic layers were dried over $\mathrm{Na}_{2} \mathrm{SO}_{4}$ and concentrated under vacuum. The remainder was purified by flash chromatography over silica gel $\left(\mathrm{CHCl}_{3} / \mathrm{MeOH}, 4: 1\right)$ to give the dihydroxy phenol $\mathbf{2 0}(0.623 \mathrm{~g}$, $92 \%$ from 18 over 2 steps) as a white solid. 
20: $\mathrm{R}_{f}=0.10\left(\mathrm{CHCl}_{3} / \mathrm{MeOH}, 4: 1\right)$. M.p. $=196-198{ }^{\circ} \mathrm{C} .{ }^{1} \mathrm{H}$ NMR $\left(600 \mathrm{MHz}, \mathrm{CDCl}_{3}\right): \delta=1.15$ $(\mathrm{td}, J=13.9,2.6 \mathrm{~Hz}, 1 \mathrm{H}, \mathrm{H}-5 \mathrm{~b}), 1.38-1.48$ (m, $1 \mathrm{H}, \mathrm{H}-6 \mathrm{~b}), 1.57$ (td, $J=12.8,4.9 \mathrm{~Hz}, 1 \mathrm{H}, \mathrm{H}-$ 15a), 1.67-1.74 (m, 2 H, H-6b and H-15b), 1.83 (br s, $1 \mathrm{H}, \mathrm{H}-14$ ), 2.09 (td, $J=12.3,3.2 \mathrm{~Hz}, 1$ H, H-16b), 2.38 (s, 3 H, NMe), 2.45 (dd, $J=12.2,3.2$ Hz, 1 H, H-16a), 2.97 (d, $J=18.1$ Hz, 1 H, H-10b), 3.09 (dd, $J=6.4,2.3$ Hz, 1 H, H-9), 3.18 (dd, $J=18.1,6.8$ Hz, 1 H, H-10a), 3.543.59 (m, 1 H, H-5a), 3.62 (dt, $J=11.7,4.0$ Hz, 1 H, H-7), 3.86 (s, 3 H, OMe), 3.90 (br s, 1 H, H-8), 6.13 (br s, $1 \mathrm{H}$, phenol), 6.64 (d, $J=8.3 \mathrm{~Hz}, 1 \mathrm{H}, \mathrm{H}-2), 6.71$ (d, $J=8.3 \mathrm{~Hz}, 1 \mathrm{H}, \mathrm{H}-1)$ ppm. ${ }^{13} \mathrm{C}$ NMR (151 MHz, $\left.\mathrm{CDCl}_{3}\right): \delta=25.5$ (t, C-10), 26.6 (t, C-6), 34.8 (t, C-5), 35.5 (s, C13), 39.6 (t, C-15), 42.3 (q, NMe) 47.2 (t, C-16), 48.7 (d, C-14), 56.1 (q, OMe), 57.6 (d, C-9), 72.5 (d, C-7), 73.4 (d, C-8), 108.8 (d, C-2), 118.2 (d, C-1) 124.5 (s, C-12), 134.1 (s, C-11), 143.7 (s, C-4), 144.5 (s, C-3) ppm. IR (ATR): v = 3572 (w), 3201 (br, w), 3019 (w), 2929 (w), 2896 (w), 2863 (w), 2834 (w), 1509 (w), 1477 (m), 1438 (m), 1321 (w), 1277 (s), 1242 (m), 1217 (m), 1138 (m), 1072 (s), 1045 (s), 917 (m), 843 (m), 816 (w), 788 (s), 762 (m), 702 (s) $\mathrm{cm}^{-1}$. ESI-MS: $\mathrm{m} / \mathrm{z}=320.3[\mathrm{M}+\mathrm{H}]^{+}, 661.4[2 \mathrm{M}+\mathrm{Na}]^{+}$. HRMS (ESI): $\mathrm{m} / \mathrm{z}$ calcd for $\mathrm{C}_{18} \mathrm{H}_{26} \mathrm{NO}_{4}$ $[\mathrm{M}+\mathrm{H}]^{+}:$320.1856; found 320.1862.

\section{Preparation of cyclohexene 22}

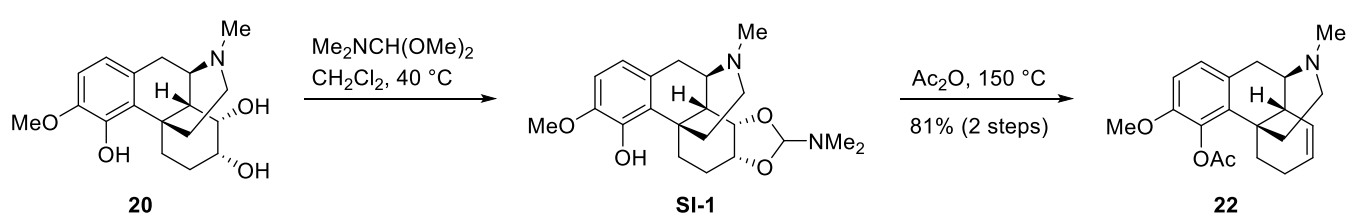

A solution of dihydroxy phenol $20(0.428 \mathrm{~g}, 1.34 \mathrm{mmol})$ in $\mathrm{CH}_{2} \mathrm{Cl}_{2}(13 \mathrm{~mL})$ was treated with $N, N$-dimethylformamide dimethyl acetal $(3.5 \mathrm{~mL}, 26 \mathrm{mmol})$, and the mixture was stirred for 20 $\mathrm{h}$ at $40{ }^{\circ} \mathrm{C}$. After cooling to room temperature and concentration of the mixture under vacuum using a rotary evaporator, the resulting crude product SI-1 was dried under high vacuum.

Crude SI-1 was dissolved in acetic anhydride $(10 \mathrm{~mL})$, and the mixture was heated to $150{ }^{\circ} \mathrm{C}$ with stirring for $2.5 \mathrm{~h}$. After cooling to $0{ }^{\circ} \mathrm{C}, 0.15 \mathrm{~N} \mathrm{HCl}(5 \mathrm{~mL})$ was added, and stirring was continued for $1 \mathrm{~h}$ at room temperature. Following concentration under vacuum, dilution with water $(10 \mathrm{~mL})$, and neutralization with $\mathrm{NaHCO}_{3}$, the mixture was extracted with $\mathrm{CH}_{2} \mathrm{Cl}_{2}(4 \times$ $10 \mathrm{~mL}$ ). The combined extracts were dried over $\mathrm{MgSO}_{4}$, the solvent was removed under vacuum, and flash chromatography of the residue over silica gel $\left(\mathrm{CHCl}_{3} / \mathrm{MeOH}, 10: 1\right)$ yielded olefin $22(0.354 \mathrm{~g}, 81 \%)$ as a slightly yellow foam. 
22: $\mathrm{R}_{f}=0.27\left(\mathrm{CHCl}_{3} / \mathrm{MeOH}, 10: 1\right) .{ }^{1} \mathrm{H} \mathrm{NMR}\left(600 \mathrm{MHz}, \mathrm{CDCl}_{3}\right): \delta=1.44-1.59(\mathrm{~m}, 1 \mathrm{H}, \mathrm{H}-$ 15a), 1.63 (dt, $J=13.0,9.1 \mathrm{~Hz}, 1 \mathrm{H}, \mathrm{H}-5 \mathrm{~b}), 1.75$ (td, $J=12.6,4.1 \mathrm{~Hz}, 1 \mathrm{H}, \mathrm{H}-15 \mathrm{~b}), 1.97-2.22$ (m, 3 H, H-6 and H-16a), 2.30 (s, 3 H, COMe), 2.43 (s, 3 H, NMe), 2.55-2.65 (m, 2 H, H-14 and H-16b), 2.66-2.83 (m, $1 \mathrm{H}, \mathrm{H}-5 \mathrm{a}), 2.76$ (dd, $J=18.1,4.5 \mathrm{~Hz}, 1 \mathrm{H}, \mathrm{H}-10 \mathrm{a}), 2.94$ (d, $J=18.1$ Hz, $1 \mathrm{H}, \mathrm{H}-10 \mathrm{~b}$ ), 3.06 (br s, $1 \mathrm{H}, \mathrm{H}-9$ ), 3.77 (s, $3 \mathrm{H}, \mathrm{OMe}$ ), 5.38 (dd, J= 9.8, $1.5 \mathrm{~Hz}, 1 \mathrm{H}, \mathrm{H}-8$ ), 5.53-5.59 (m, $1 \mathrm{H}, \mathrm{H}-7), 6.78$ (d, $J=8.3 \mathrm{~Hz}, 1 \mathrm{H}, \mathrm{H}-2), 6.93$ (d, $J=8.3 \mathrm{~Hz}, 1 \mathrm{H}, \mathrm{H}-1)$ ppm. ${ }^{13} \mathrm{C}$ NMR (151 MHz, $\mathrm{CDCl}_{3}$ ): $\delta=21.1$ (q, $\mathrm{COCH}_{3}$ ), 23.8 (t, C-6), 24.2 (t, C-10), 33.0 (t, C-5), 36.1 (s, C-13), 37.3 (t, C-15), 42.5 (q, NMe), 46.1 (d, C-14), 48.2 (t, C-16), 55.9 (q, OMe), 57.2 (d, C-9), 110.0 (d, C-2), 125.4 (d, C-1), 127.2 (d, C-8), 129.1 (d, C-7), 130.9 (s, C-11), 132.0 (s, C-12) 139.0 (s, C-4) 149.3 (s, C-3), 168.8 (s, $\boldsymbol{C O C H}_{3}$ ) ppm. IR (ATR): v= 3013 (w), 2902 (w), 2839 (w), 2799 (w), 1762 (m), 1509 (w), 1482 (m), 1458 (m), 1434 (m), 1365 (m), 1278 (s), 1211 (s), 1195 (s), 1177 (s), 1150 (s), 1052 (s), 1022 (s), 950 (w), 920 (w), 878 (w), 799 (m), $670(\mathrm{~m}) \mathrm{cm}^{-1}$. ESI-MS: $\mathrm{m} / \mathrm{z}=328.2[\mathrm{M}+\mathrm{H}]^{+}, 655.0[2 \mathrm{M}+\mathrm{H}]^{+}$. HRMS (ESI): m/z calcd for $\mathrm{C}_{20} \mathrm{H}_{26} \mathrm{NO}_{3}[\mathrm{M}+\mathrm{H}]^{+}:$328.1907; found 328.1909.

\section{Preparation of ethyl carbamate 23}

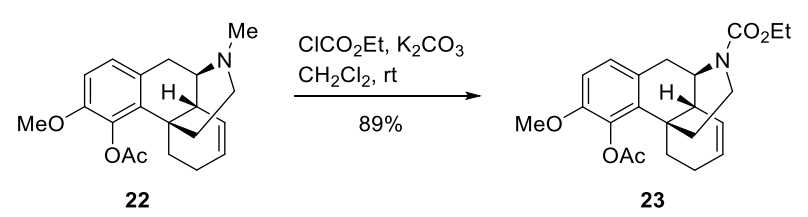

Ethyl chloroformate $(0.19 \mathrm{~mL}, 2.0 \mathrm{mmol})$ was added to a mixture of amine $22(0.133 \mathrm{~g}, 0.406$ mmol) and $\mathrm{K}_{2} \mathrm{CO}_{3}(0.280 \mathrm{~g}, 2.03 \mathrm{mmol})$ in $\mathrm{CH}_{2} \mathrm{Cl}_{2}(4 \mathrm{~mL})$. After stirring the mixture at room temperature for $8 \mathrm{~h}$, further ethyl chloroformate $(0.19 \mathrm{~mL}, 2.0 \mathrm{mmol})$ was added. Stirring was continued for $21 \mathrm{~h}$ at room temperature, and then the mixture was filtered over silica gel in order to remove the solids, and the filtrate was concentrated under vacuum. Purification of the remainder by flash chromatography over silica gel $\left(\mathrm{CH}_{2} \mathrm{Cl}_{2} / \mathrm{Et}_{2} \mathrm{O}, 20: 1\right)$ afforded ethyl carbamate 23 ( $0.140 \mathrm{~g}, 0.363 \mathrm{mmol}, 89 \%)$ as a white powder.

23: $\mathrm{R}_{f}=0.20\left(\mathrm{CH}_{2} \mathrm{Cl}_{2} / \mathrm{Et}_{2} \mathrm{O}, 20: 1\right)$. M.p. $=152-153{ }^{\circ} \mathrm{C} .{ }^{1} \mathrm{H}$ NMR $\left(600 \mathrm{MHz}, \mathrm{CDCl}_{3}\right): \delta=1.21$ (major amide rotamer) (t, $J=7.0 \mathrm{~Hz}, 2 \mathrm{H}), 1.29$ (minor amide rotamer) (t, $J=7.0 \mathrm{~Hz}, 1 \mathrm{H}$ ), 1.51-1.64 (m, 2 H), 2.02 (br s, 2 H), 2.31 (s, 3 H), 2.41 (br s, 1 H), 2.48-2.87 (m, 3 H), 3.03$3.18(\mathrm{~m}, 1 \mathrm{H}), 3.77(\mathrm{~s}, 3 \mathrm{H}), 3.82$ (major amide rotamer) (d, $J=14.3 \mathrm{~Hz}, 0.6 \mathrm{H}$ ), 3.93 (minor amide rotamer) $(\mathrm{d}, J=11.3 \mathrm{~Hz}, 0.4 \mathrm{H}), 4.05-4.21(\mathrm{~m}, 2 \mathrm{H}), 4.38$ (major amide rotamer) (br s, $0.4 \mathrm{H}), 4.51$ (minor amide rotamer) (br s, $0.6 \mathrm{H}) 5.34-5.44$ (m, $1 \mathrm{H}) 5.61$ (br s, $1 \mathrm{H}), 6.79$ (d, $J$ 
$=8.3 \mathrm{~Hz}, 1 \mathrm{H}), 6.85-6.95(\mathrm{~m}, 1 \mathrm{H}) \mathrm{ppm} .{ }^{13} \mathrm{C} \mathrm{NMR}\left(151 \mathrm{MHz}, \mathrm{CDCl}_{3}\right): \delta=14.6,14.8,21.0$, 23.5, 31.3, 31.7, 32.8, 32.9, 36.3, 36.7, 36.9, 39.3, 45.0, 49.3, 49.5, 55.9, 61.1, 61.2, 110.4, 126.1, 126.5, 129.6, 129.9, 130.2, 131.1, 139.2, 149.47, 149.43, 155.9, 156.2, 168.7, 168.9 ppm. IR (ATR): v = 3015 (w), 2997 (w), $2958(w), 2931(w), 2890(w), 2836(w), 1764(\mathrm{~m}), 1691$ (s), $1486(\mathrm{~m}), 1415$ (s), 1370 (m), 1335 (m), 1299 (m), 1277 (s), 1244 (m), 1212 (s), 1176 (s), 1129 (s), 1098 (s), 1056 (s), 1023 (s), 948 (m), 912 (m), 869 (w), 832 (m), 789 (m), 765 (m), $682(\mathrm{~m}) \mathrm{cm}^{-1}$. ESI-MS: $\mathrm{m} / \mathrm{z}=386.4[\mathrm{M}+\mathrm{H}]^{+}, 771.6[2 \mathrm{M}+\mathrm{H}]^{+}, 793.5[2 \mathrm{M}+\mathrm{Na}]^{+}$. HRMS (ESI): $\mathrm{m} / \mathrm{z}$ calcd for $\mathrm{C}_{22} \mathrm{H}_{28} \mathrm{NO}_{5}[\mathrm{M}+\mathrm{H}]^{+}:$386.1962; found 386.1964.

\section{Preparation of cyclohexenone 24 by allylic oxidation}

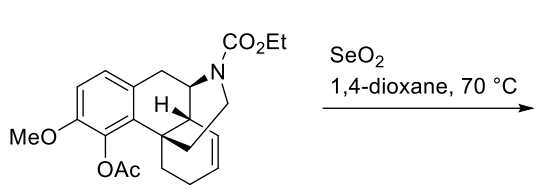

23

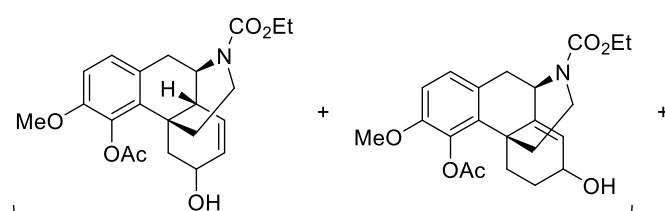

SI-2

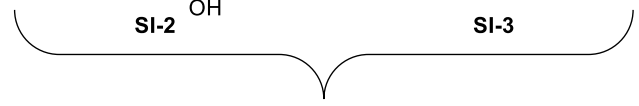

$(35 \%)$

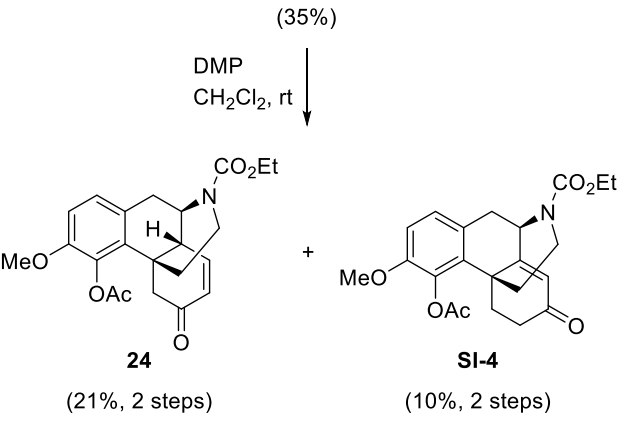

A mixture of ethyl carbamate 23 ( $0.186 \mathrm{~g}, 0.483 \mathrm{mmol})$, selenium dioxide $(0.125 \mathrm{~g}, 1.13 \mathrm{mmol})$, and sand $(0.568 \mathrm{~g})$ in 1,4-dioxane $(9 \mathrm{~mL})$ was heated to $70^{\circ} \mathrm{C}$ oil bath temperature with stirring for $8 \mathrm{~h}$. After addition of another portion of selenium dioxide $(0.125 \mathrm{~g}, 1.13 \mathrm{mmol})$, heating at $70{ }^{\circ} \mathrm{C}$ oil bath temperature was continued for further $19 \mathrm{~h}$. The mixture was cooled to $0{ }^{\circ} \mathrm{C}$, treated with saturated aqueous $\mathrm{NaHCO}_{3}$ solution $(3 \mathrm{~mL})$, and stirred for $45 \mathrm{~min}$. Water $(3 \mathrm{~mL})$ was added, and the mixture was extracted with $\mathrm{CH}_{2} \mathrm{Cl}_{2}(4 \times 6 \mathrm{~mL})$. The combined extracts were dried over $\mathrm{MgSO}_{4}$, and the solvent was removed under vacuum. The residue was subjected to flash chromatography over silica gel $\left(\mathrm{CH}_{2} \mathrm{Cl}_{2} / \mathrm{Et}_{2} \mathrm{O}, 1: 1\right)$ to give two fractions of the same molecular mass according to MS analysis. Fraction $1\left[\mathrm{R}_{f}=0.31\left(\mathrm{CH}_{2} \mathrm{Cl}_{2} / \mathrm{Et}_{2} \mathrm{O}, 1: 1\right)\right]$ was identified as the tertiary allylic alcohol $27(40.0 \mathrm{mg}, 21 \%)$, and fraction $2\left[\mathrm{R}_{f}=0.17\right.$ $\left.\left(\mathrm{CH}_{2} \mathrm{Cl}_{2} / \mathrm{Et}_{2} \mathrm{O}, 1: 1\right)\right]$ contained the two regioisomeric allylic alcohols $\mathbf{S I - 2}$ and $\mathbf{S I - 3}$ (67.0 mg, $35 \%$ ) of unknown relative configuration at the carbinol center, which could not be separated. 
Fraction 2 (SI-2 and SI-3, $67.0 \mathrm{mg}, 0.167 \mathrm{mmol}$ ) obtained as described above was dissolved in $\mathrm{CH}_{2} \mathrm{Cl}_{2}(5 \mathrm{~mL})$, and Dess-Martin periodinane $(0.142 \mathrm{~g}, 0.334 \mathrm{mmol})$ was added at room temperature. After stirring at this temperature for $5 \mathrm{~h}$, the reaction mixture was cooled to $0{ }^{\circ} \mathrm{C}$, and a mixture of saturated aqueous $\mathrm{NaHCO}_{3}$ solution $(3 \mathrm{~mL})$ and $\mathrm{Na}_{2} \mathrm{SO}_{3}(42 \mathrm{mg}$ ) was added. The resulting mixture was extracted with $\mathrm{CH}_{2} \mathrm{Cl}_{2} / \mathrm{Et}_{2} \mathrm{O}(1: 2,4 \times 5 \mathrm{~mL})$ and dried over $\mathrm{MgSO}_{4}$. After removal of the solvent under vacuum, the crude product was purified by flash chromatography over silica gel $\left(\mathrm{CH}_{2} \mathrm{Cl}_{2} / \mathrm{Et}_{2} \mathrm{O}, 1: 1\right)$ to give the two unsaturated ketones 24 (40.2 mg, 21\% from 23 over 2 steps) and SI-4 (20.0 mg, 10\% from 23 over 2 steps).

24: $\mathrm{R}_{f}=0.32\left(\mathrm{CH}_{2} \mathrm{Cl}_{2} / \mathrm{Et}_{2} \mathrm{O}, 1: 1\right) .{ }^{1} \mathrm{H} \mathrm{NMR}\left(600 \mathrm{MHz}, \mathrm{CDCl}_{3}\right): \delta=1.22-1.35(\mathrm{~m}, 3 \mathrm{H}), 1.64-$ $1.80(\mathrm{~m}, 2 \mathrm{H}), 2.36$ (s, $3 \mathrm{H}), 2.44$ (d, $J=15.8 \mathrm{~Hz}, 1 \mathrm{H}), 2.55-2.70(\mathrm{~m}, 1 \mathrm{H}), 2.73-2.83$ (m, 2 H), $3.09(\mathrm{dd}, J=18.1,5.3 \mathrm{~Hz}, 1 \mathrm{H}), 3.71-3.80(\mathrm{~m}, 1 \mathrm{H}), 3.74(\mathrm{~s}, 3 \mathrm{H}), 3.86$ (major amide rotamer) (d, $J=13.2 \mathrm{~Hz}, 0.6 \mathrm{H}), 3.98$ (minor amide rotamer) $(\mathrm{d}, J=13.2 \mathrm{~Hz}, 0.4 \mathrm{H}), 4.10-4.23$ $(\mathrm{m}, 2 \mathrm{H}), 4.66$ (minor amide rotamer) (br s, $0.4 \mathrm{H}), 4.79$ (major amide rotamer) (br s, $0.6 \mathrm{H})$, $5.95(\mathrm{~d}, J=9.8 \mathrm{~Hz}, 1 \mathrm{H}), 6.63-6.71(\mathrm{~m}, 1 \mathrm{H}), 6.79(\mathrm{~d}, J=8.3 \mathrm{~Hz}, 1 \mathrm{H}), 6.86-6.92(\mathrm{~m}, 1 \mathrm{H})$ ppm. ${ }^{13} \mathrm{C} \mathrm{NMR}\left(151 \mathrm{MHz}, \mathrm{CDCl}_{3}\right): \delta=14.6,14.8,21.1,31.9,32.3,36.9,37.0,37.9,38.2,40.8$, 45.7, 47.9, 48.3, 49.28, 49.29, 49.4, 49.5, 56.0, 61.5, 111.2, 126.1, 126.2, 128.5, 129.0, 131.5, 131.57, 131.6, 139.5, 147.3, 147.6, 150.06, 150.11, 155.5, 156.0, 197.1 ppm. IR (ATR): $v=$ 3019 (w), 2982 (w), 2928 (w), 2865 (w), 2840 (w), 1781 (m), 1696 (w), 1675 (s), 1476 (m), 1458 (m), 1372 (m), 1337 (m), 1300 (m), 1273 (m), 1230 (m), 1175 (s), 1131 (m), 1092 (m), 1057 (m), 1026 (m), 957 (w), 894 (m), 791 (m), $773(\mathrm{~m}) \mathrm{cm}^{-1}$. ESI-MS: m/z = $400.5[\mathrm{M}+\mathrm{H}]^{+}$, $821.6[2 \mathrm{M}+\mathrm{Na}]^{+}$. HRMS (ESI): $\mathrm{m} / \mathrm{z}$ calcd for $\mathrm{C}_{22} \mathrm{H}_{26} \mathrm{NO}_{6}[\mathrm{M}+\mathrm{H}]^{+}: 400.1755$; found 400.1761, $\mathrm{m} / \mathrm{z}$ calcd for $[\mathrm{M}+\mathrm{Na}]^{+}:$422.1574; found 422.1582 .

SI-4: $\mathrm{R}_{f}=0.44\left(\mathrm{CH}_{2} \mathrm{Cl}_{2} / \mathrm{Et}_{2} \mathrm{O}, 1: 1\right) .{ }^{1} \mathrm{H}$ NMR $\left(600 \mathrm{MHz}, \mathrm{CDCl}_{3}\right): \delta=1.19-1.32(\mathrm{~m}, 3 \mathrm{H}), 1.95$ (br s, 2 H), 2.33 (s, 3 H, COMe) 2.35-2.43 (m, 2 H), 2.43-2.51 (m, 1 H), 2.85 (br s, 2 H), 3.00$3.31(\mathrm{~m}, 2 \mathrm{H}), 3.79$ (s, $3 \mathrm{H}, \mathrm{OMe}) 3.90$ (major amide rotamer) (d, $J=12.4 \mathrm{~Hz}, 0.6 \mathrm{H}), 4.01$ (minor amide rotamer) $(\mathrm{d}, J=12.4 \mathrm{~Hz}, 0.4 \mathrm{H}), 4.06-4.24(\mathrm{~m}, 2 \mathrm{H}), 4.92$ (minor amide rotamer) (br s, $0.4 \mathrm{H}), 5.04$ (major amide rotamer) (br s, $0.6 \mathrm{H}), 5.95-6.07(\mathrm{~m}, 1 \mathrm{H}), 6.86$ (d, $J=8.3 \mathrm{~Hz}$, $1 \mathrm{H}), 6.91-7.05$ (m, $1 \mathrm{H}) \mathrm{ppm} .{ }^{13} \mathrm{C} \mathrm{NMR}\left(151 \mathrm{MHz}, \mathrm{CDCl}_{3}\right): \delta=14.5,14.7,21.0,31.5,34.7$, $36.5,38.7,39.0,39.2,40.0,53.6,53.8,56.1,61.6,61.7,111.4,122.2,126.5,126.6,128.6$, 128.9, 132.1, 138.4, 150.2, 155.2, 155.5, 160.4, 168.7, 198.8 ppm. IR (ATR): $v=2977(w)$, 2938 (w), 2841 (w), 2840 (w), 1767 (m), 1679 (s), 1483 (m), 1426 (m), 1372 (m), 1303 (m), 
1279 (s), 1230 (m), 1186 (s), 1164 (s), 1124 (m), 1096 (m), 1050 (m), 1034 (m), 921 (m), 874 (m), $770(\mathrm{~m}) \mathrm{cm}^{-1}$. ESI-MS: m/z = $400.4[\mathrm{M}+\mathrm{H}]^{+}, 422.3[\mathrm{M}+\mathrm{Na}]^{+}$. HRMS (ESI): m/z calcd for $\mathrm{C}_{22} \mathrm{H}_{26} \mathrm{NO}_{6}[\mathrm{M}+\mathrm{H}]^{+}:$400.1755; found $400.1760, \mathrm{~m} / \mathrm{z}$ calcd for $\mathrm{C}_{22} \mathrm{H}_{25} \mathrm{NNaO}_{6}[\mathrm{M}+\mathrm{Na}]^{+}$: 422.1574; found 422.1563.

27: $\mathrm{R}_{f}=0.31\left(\mathrm{CH}_{2} \mathrm{Cl}_{2} / \mathrm{Et}_{2} \mathrm{O}, 1: 1\right) .{ }^{1} \mathrm{H}$ NMR $\left(600 \mathrm{MHz}, \mathrm{CDCl}_{3}\right): \delta=1.20-1.37(\mathrm{~m}, 3 \mathrm{H}), 1.92-$ 2.27 (m, 5 H), 2.32 (s, 3 H), 2.44-2.72 (m, 2 H), 2.75-2.88 (m, 1 H), 3.21 (dd, J= 17.7, $5.3 \mathrm{~Hz}$, $1 \mathrm{H}$ ), 3.77 (s, $3 \mathrm{H}), 3.76-3.97(\mathrm{~m}, 1 \mathrm{H}), 4.13(\mathrm{~m}, 2 \mathrm{H}), 4.29$ (minor amide rotamer) (br s, 0.4 H), 4.41 (br s, 0,6 H), 5.64 (br s, $1 \mathrm{H}), 5.78$ (d, J=7.5 Hz, $1 \mathrm{H}), 6.81$ (d, J = 8.7 Hz, $1 \mathrm{H}), 6.84$ 6.91 (m, 1 H) ppm. IR (ATR): v = 3401 (br, w), 2979 (w), 2940 (w), 2900 (w), 2839 (w), 1765 (m), 1676 (s), 1655 (m), 1484 (m), 1458 (m), 1431 (s), 1372 (m), 1326 (w), 1298 (m), 1277 (s), 1212 (s), 1177 (s), 1131 (s), 1096 (m), 1035 (s), 1010 (s), 954 (m), 912 (w), 835 (m), 798 (m), $768(\mathrm{~m}) \mathrm{cm}^{-1}$. HRMS (ESI): $\mathrm{m} / \mathrm{z}$ calcd for $\mathrm{C}_{22} \mathrm{H}_{28} \mathrm{NO}_{6}[\mathrm{M}+\mathrm{H}]^{+}: 402.1911$; found $402.1916, \mathrm{~m} / \mathrm{z}$ calcd for $\mathrm{C}_{22} \mathrm{H}_{27} \mathrm{NNaO}_{6}[\mathrm{M}+\mathrm{Na}]^{+}$: 424.1731; found 424.1740.

\section{Reduction of carbamate 27 to give $N$-methyl phenol SI-5}

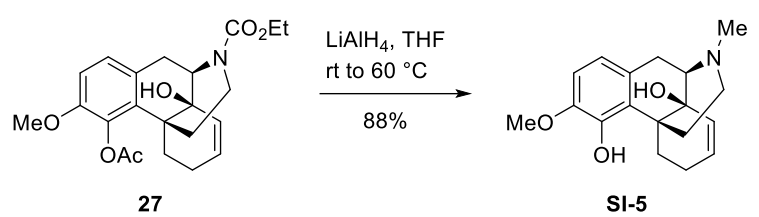

A solution of tertiary allylic alcohol $27(74.4 \mathrm{mg}, 0.186 \mathrm{mmol}$, dried under high vacuum) in dry THF $(5 \mathrm{~mL})$ was treated with a solution of lithium aluminum hydride in diethyl ether $(0.56 \mathrm{~mL}$, $1 \mathrm{M})$ at room temperature. Stirring was continued for $30 \mathrm{~min}$ at room temperature and $4 \mathrm{~h}$ at 60 ${ }^{\circ} \mathrm{C}$ oil bath temperature. The mixture was then cooled to $0{ }^{\circ} \mathrm{C}$, and $\mathrm{Na}_{2} \mathrm{SO}_{4}$ decahydrate (Glauber's salt) $(0.180 \mathrm{~g})$ was added in order to destroy excess hydride. After the first gas evolution had ceased, the cooling bath was removed, and the reaction mixture was allowed to warm to room temperature. Subsequently, the mixture was filtered over silica gel and eluted with $\mathrm{CH}_{2} \mathrm{Cl}_{2} /$ methanol (1:1). The solvent was removed under vacuum, and the residue was purified by flash chromatography over silica gel $\left(\mathrm{CH}_{2} \mathrm{Cl}_{2} / \mathrm{MeOH}, 85: 15\right.$ containing $0.4 \%$ concentrated ammonia) to give the $N$-methyl phenol SI-5 (49.2 mg, 88\%) as a white powder.

SI-5: $\mathrm{R}_{f}=0.15\left(\mathrm{CH}_{2} \mathrm{Cl}_{2} / \mathrm{MeOH}, 4: 1\right) . \mathrm{M} . \mathrm{p} .=166-169{ }^{\circ} \mathrm{C} .{ }^{1} \mathrm{H} \mathrm{NMR}\left(600 \mathrm{MHz}, \mathrm{CDCl}_{3}\right): \delta=$ 1.54-1.59 (m, 1 H, H-15), 1.91-2.00 (m, 1 H, H-6), 2.01-2.15 (m, 4 H, H-6/H-15/H-16/H-5), 2.36 (s, 3 H, NMe), 2.39-2.45 (m, 1 H, H-16), 2.76-2.84 (m, 1 H, H-10a), 2.80 (br s, 1 H, H- 
9), 2.87 (dd, $J=12.8,4.9 \mathrm{~Hz}, 1 \mathrm{H}, \mathrm{H}-5), 2.98-3.07$ (m, $1 \mathrm{H}, \mathrm{H}-10 \mathrm{~b}$ ), 3.85 (s, $3 \mathrm{H}, \mathrm{OMe}$ ), 4.43 (br s, $1 \mathrm{H}, \mathrm{OH}$ ), 5.51 (d, J = 9.8 Hz, $1 \mathrm{H}, \mathrm{H}-8), 5.77-5.84$ (m, $1 \mathrm{H}, \mathrm{H}-9), 5.93$ (s, $1 \mathrm{H}$, phenol), $6.55(\mathrm{~d}, J=8.3 \mathrm{~Hz}, 1 \mathrm{H}, \mathrm{H}-1), 6.67$ (dd, $J=8.3 \mathrm{~Hz}, 1 \mathrm{H}, \mathrm{H}-2) \mathrm{ppm} .{ }^{13} \mathrm{C} \mathrm{NMR}(151 \mathrm{MHz}$, $\left.\mathrm{CDCl}_{3}\right): \delta=24.6(\mathrm{t}, \mathrm{C}-6), 24.9$ (t, C-10), $25.4(\mathrm{t}, \mathrm{C}-5), 31.2(\mathrm{t}, \mathrm{C}-15), 40.2(\mathrm{~s}, \mathrm{C}-13), 42.5(\mathrm{q}$, NMe), 47.3 (t, C-16), 56.1 (q, OMe), 62.1 (d, C-9), 68.0 (s, C-14), 108.4 (d, C-1), 117.8 (d, C2), 125.6 (s, C-12), 128.5 (d, C-8), 131.0 (s, C-11), 134.4 (d, C-7), 144.2 (s, C-4), 144.3 (s, C3) ppm. IR (ATR): v = 3493 (m), $3429(w), 2931$ (w), $2911(w), 2892(w), 2866(w), 2827(w)$, 1486 (m), 1451 (m), 1434 (m), 1403 (w), 1376 (w), 1356 (m), 1327 (m), 1267 (s), 1220 (s), 1120 (m), 1103 (m), 1065 (s), 1011 (s), 963 (m), 944 (m), 899 (m), 833 (m), 801 (m), 781 (m),707 (m), $688(\mathrm{~m}), 663(\mathrm{~m}) \mathrm{cm}^{-1}$. ESI-MS: m/z = $302.1[\mathrm{M}+\mathrm{H}]^{+}$. HRMS (ESI): m/z calcd for $\mathrm{C}_{18} \mathrm{H}_{24} \mathrm{NO}_{3}[\mathrm{M}+\mathrm{H}]^{+}:$302.1751; found 302.1752.

The relative configuration at C-14 of SI-5 was elucidated by 2D NMR measurements. Thus, the vinylic proton at C-8 showed significant NOEs with H-9 and H-10a. The latter interaction is only possible with a $\beta$ orientation of the hydroxy group at $\mathrm{C}-14$.

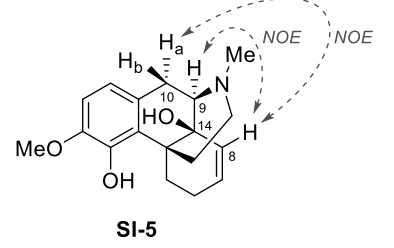

\section{Preparation of dioxolane 25}

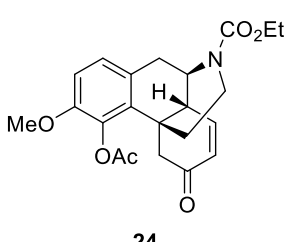

24

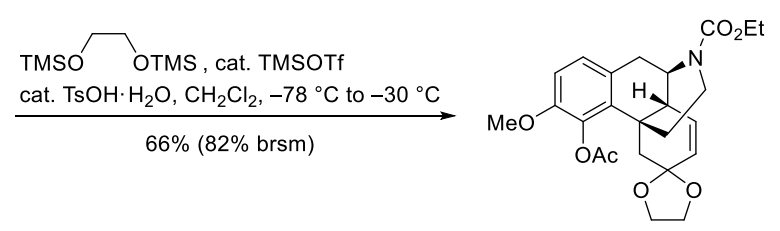

25

A mixture of enone 24 (0.200 g, $0.501 \mathrm{mmol})$, 1,2-bis(trimethylsiloxy)ethane (0.134 g, 0.649 $\mathrm{mmol})$ and $\mathrm{TsOH} \cdot \mathrm{H}_{2} \mathrm{O}(2.9 \mathrm{mg}, 0.015 \mathrm{mmol}$, dried under high vacuum $)$ in dry $\mathrm{CH}_{2} \mathrm{Cl}_{2}(3.5 \mathrm{~mL})$ was stirred at room temperature for $30 \mathrm{~min}$. After cooling the mixture to $-78{ }^{\circ} \mathrm{C}$, trimethylsilyl triflate $(7.8 \mathrm{mg}, 0.035 \mathrm{mmol})$ was added, stirring was continued for $24 \mathrm{~h}$ at $-30{ }^{\circ} \mathrm{C}$, and then pyridine $(0.05 \mathrm{~mL})$ was added at this temperature followed by dilution with diethyl ether $(5$ $\mathrm{mL})$ and treatment with saturated aqueous $\mathrm{NaHCO}_{3}$ solution $(3 \mathrm{~mL})$. The mixture was allowed to warm to room temperature and extracted with $\mathrm{Et}_{2} \mathrm{O} / \mathrm{CH}_{2} \mathrm{Cl}_{2}(2: 1,3 \times 3 \mathrm{~mL})$. The combined organic layers were washed with saturated aqueous $\mathrm{NaCl}$ solution $(3 \mathrm{~mL})$ and dried over MgSO4. After removal of the solvent under vacuum, the residue was purified by flash 
chromatography over silica gel $\left(\mathrm{CH}_{2} \mathrm{Cl}_{2} / \mathrm{Et}_{2} \mathrm{O}, 3: 7\right)$ to give starting material 24 (38.6 mg, 19\%) and dioxolane 25 (146.5 $\mathrm{mg}, 66 \%, 82 \%$ brsm) as a white foam.

25: $\mathrm{R}_{f}=0.40\left(\mathrm{CH}_{2} \mathrm{Cl}_{2} / \mathrm{Et}_{2} \mathrm{O}, 3: 7\right) .{ }^{1} \mathrm{H} \mathrm{NMR}\left(600 \mathrm{MHz}, \mathrm{CDCl}_{3}\right): \delta=1.20$ (major amide rotamer) (t, $J=7.2 \mathrm{~Hz}, 1.8 \mathrm{H}$ ), 1.28 (minor amide rotamer) (t, $J=7.0 \mathrm{~Hz}, 1.2 \mathrm{H}), 1.52-1.65(\mathrm{~m}, 2 \mathrm{H})$, 1.69 (br s, $1 \mathrm{H}), 1.76$ (d, $J=13.6 \mathrm{~Hz}, 1 \mathrm{H}), 2.32$ (s, $3 \mathrm{H}), 2.38$ (br s, $1 \mathrm{H}), 2.53-2.74(\mathrm{~m}, 2 \mathrm{H})$, $3.06(\mathrm{dd}, J=17.7,4.9 \mathrm{~Hz}, 1 \mathrm{H}), 3.27$ (d, $J=13.6 \mathrm{~Hz}, 1 \mathrm{H}), 3.73-3.82(\mathrm{~m}, 2 \mathrm{H}), 3.76(\mathrm{~s}, 3 \mathrm{H})$, 3.86-4.04 (m, $3 \mathrm{H}$ ), 4.06-4.20 (m, $2 \mathrm{H}$ ), 4.47 (minor amide rotamer) (br s, 0.4 H), 4.59 (major amide rotamer) (br s, $0.6 \mathrm{H}), 5.53(\mathrm{~d}, J=9.0 \mathrm{~Hz}, 1 \mathrm{H}), 5.70-5.77(\mathrm{~m}, 1 \mathrm{H}), 6.81(\mathrm{~d}, J=8.7 \mathrm{~Hz}$, $1 \mathrm{H}), 6.84-6.91(\mathrm{~m}, 1 \mathrm{H}) \mathrm{ppm} .{ }^{13} \mathrm{C} \mathrm{NMR}\left(151 \mathrm{MHz}, \mathrm{CDCl}_{3}\right)$ major amide rotamer: $\delta=14.5$ (q), $21.1(\mathrm{q}), 31.5(\mathrm{t}), 37.1(\mathrm{t}), 37.6(\mathrm{~s}), 38.8(\mathrm{t}), 41.8(\mathrm{t}), 44.5(\mathrm{~d}), 48.7$ (d), $56.0(\mathrm{q}, \mathrm{OMe}), 61.2$ (t), $64.2(\mathrm{t}), 65.1(\mathrm{t}), 105.1$ (s), 111.0 (d), 126.2 (d), 128.6 (s), 129.5 (d), 129.9 (s), 131.4 (d), $140.4(\mathrm{~s}), 149.2(\mathrm{~s}), 156.2(\mathrm{~s}), 168.9(\mathrm{~s})$ ppm; minor amide rotamer: $14.8(\mathrm{q}), 21.1(\mathrm{q}), 32.0(\mathrm{t})$, $37.0(\mathrm{t}), 37.6(\mathrm{~s}), 38.6(\mathrm{t}), 41.8(\mathrm{t}), 44.5(\mathrm{~d}), 49.0(\mathrm{~d}), 56.0(\mathrm{q}, \mathrm{OMe}), 61.3(\mathrm{t}), 64.2(\mathrm{t}), 65.1(\mathrm{t})$, 105.1 (s), 111.0 (d), 126.0 (d), 128.3 (s), 129.7 (d), 129.9 (s), 131.3 (d), 140.4 (s), 149.3 (s), 155.7 (s), 168.9 (s) ppm. IR (ATR): v = 2955 (w), 2914 (w), 2880 (w), 1762 (m), 1687 (s), 1653 (m), 1485 (m), 1473 (m), 1459 (m), 1419 (m), 1317 (m), 1275 (s), 1212 (s), 1189 (s), 1130 (s), 1073 (s), 1053 (s), 1018 (s), 997 (s), 946 (m), 802 (m), 769 (m) cm ${ }^{-1}$. ESI-MS: m/z = 444.3 $[\mathrm{M}+\mathrm{H}]^{+}$. HRMS (ESI): $\mathrm{m} / \mathrm{z}$ calcd for $\mathrm{C}_{24} \mathrm{H}_{30} \mathrm{NO}_{7}[\mathrm{M}+\mathrm{H}]^{+}: 444.2017$; found 444.2021, m/z calcd for $\mathrm{C}_{24} \mathrm{H}_{29} \mathrm{NNaO}_{7}[\mathrm{M}+\mathrm{Na}]^{+}$: 466.1836; found 466.1840.

\section{Preparation of acetal 26}

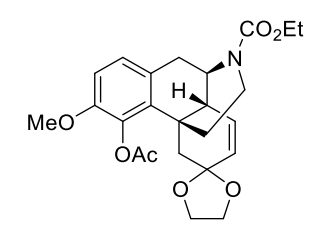

25

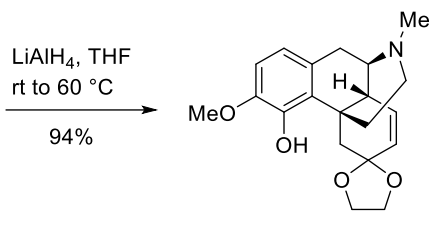

26

A solution of dioxolane 25 ( $66.5 \mathrm{mg}, 0.15 \mathrm{mmol}$, dried under high vacuum) in dry THF (4.5 $\mathrm{mL})$ was treated with a solution of lithium aluminum hydride in diethyl ether $(0.45 \mathrm{~mL}, 1 \mathrm{M})$ at room temperature. Stirring was continued for $30 \mathrm{~min}$ at room temperature and $2.5 \mathrm{~h}$ at $60{ }^{\circ} \mathrm{C}$ oil bath temperature. The mixture was then cooled to $0{ }^{\circ} \mathrm{C}$, and $\mathrm{Na}_{2} \mathrm{SO}_{4}$ decahydrate (Glauber's salt) (0.145 g) was added in order to destroy excess hydride. After the first gas evolution had ceased, the cooling bath was removed, and the reaction mixture was allowed to warm to room temperature. Subsequently, the mixture was filtered over silica gel and eluted with $\mathrm{CHCl}_{3} / \mathrm{MeOH}(3: 2)$. The solvent was removed under vacuum, and the residue was purified by 
flash chromatography over silica gel $\left(\mathrm{CHCl}_{3} / \mathrm{MeOH}, 10: 1\right)$ to give acetal $26(48.4 \mathrm{mg}, 94 \%)$ as a slightly yellow solid.

26: $\mathrm{R}_{f}=0.11\left(\mathrm{CHCl}_{3} / \mathrm{MeOH}, 10: 1\right)$. M.p. $=171-174{ }^{\circ} \mathrm{C} .{ }^{1} \mathrm{H} \mathrm{NMR}\left(600 \mathrm{MHz}, \mathrm{CDCl}_{3}\right): \delta=1.70$ (d, $J=13.6 \mathrm{~Hz}, 1 \mathrm{H}, \mathrm{H}-5 \mathrm{~b}), 1.80$ (td, $J=12.6,4.7 \mathrm{~Hz}, 1 \mathrm{H}, \mathrm{H}-15 \mathrm{a}), 1.92$ (d, $J=12.6 \mathrm{~Hz}, 1 \mathrm{H}$, H-15b), 2.08 (td, $J=12.2,3.4$ Hz, 1 H, H-16b), 2.44 (s, 3 H, NMe), 2.57-2.65 (m, 2 H, H-14 and H-16b), 2.74 (dd, $J=18.1,4.7 \mathrm{~Hz}, 1 \mathrm{H}, \mathrm{H}-10 \mathrm{a}), 2.91$ (d, $J=18.1 \mathrm{~Hz}, 1 \mathrm{H}, \mathrm{H}-10 \mathrm{~b}), 3.16$ (t, $J=3.6 \mathrm{~Hz}, 1 \mathrm{H}, \mathrm{H}-9), 3.67(\mathrm{dd}, J=13.6,1.5 \mathrm{~Hz}, 1 \mathrm{H}, \mathrm{H}-5 \mathrm{a}), 3.76(\mathrm{dd}, J=14.3,7.2 \mathrm{~Hz}, 1 \mathrm{H}$, $\mathrm{OCH}_{2} \mathrm{CH}_{2} \mathrm{O}$ ), 3.83 (s, $\left.3 \mathrm{H}, \mathrm{OMe}\right), 3.88-3.92\left(\mathrm{~m}, 2 \mathrm{H}, \mathrm{OCH}_{2} \mathrm{CH}_{2} \mathrm{O}\right), 3.98$ (dt, $J=7.3,5.0 \mathrm{~Hz}, 1$ $\mathrm{H}, \mathrm{OCH}_{2} \mathrm{CH}_{2} \mathrm{O}$ ), 5.45-5.51 (m, $\left.1 \mathrm{H}, \mathrm{H}-7\right), 5.73$ (dd, $\left.J=10.0,1.3 \mathrm{~Hz}, 1 \mathrm{H}, \mathrm{H}-8\right), 5.99$ (s, $1 \mathrm{H}$, phenol), 6.54 (d, $J=8.3 \mathrm{~Hz}, 1 \mathrm{H}, \mathrm{H}-1), 6.67$ (d, $J=8.3 \mathrm{~Hz}, 1 \mathrm{H}, \mathrm{H}-2)$ ppm. ${ }^{13} \mathrm{C}$ NMR (151 $\mathrm{MHz}, \mathrm{CDCl}_{3}$ ): $\delta=24.6$ (t, C-10), 36.1 (t, C-15), 37.3 (s, C-13), 41.0 (t, C-5), 42.3 (q, NMe), 45.2 (d, C-14), 48.0 (t, C-16), 56.1 (q, OMe), 57.0 (d, C-9), 64.0 (t, $\mathrm{OCH}_{2} \mathrm{CH}_{2} \mathrm{O}$ ), 65.0 (t, $\mathrm{OCH}_{2} \mathrm{CH}_{2} \mathrm{O}$ ), 105.6 (s, C-6), 108.8 (d, C-2), 118.3 (d, C-1), 123.9 (s, C-12), 129.0 (d, C-7), 129.6 (s, C-11), 132.7 (d, C-8), 144.4 (s, C-3), 145.2 (s, C-4) ppm. IR (ATR): v = 2954 (w), 2914 (w), 2883 (w), 2842 (w), 2832 (w), 2804 (w), 1486 (m), 1472 (m), 1459 (m), 1440 (m), 1396 (m), 1277 (s), 1235 (m), 1218 (s), 1129 (m), 1075 (s), 1052 (s), 1012 (s), 998 (s), 924 (m), 849 (w), 805 (s), 731 (s) $\mathrm{cm}^{-1}$. ESI-MS: m/z = $344.4[\mathrm{M}+\mathrm{H}]^{+}$. HRMS (ESI): m/z calcd for $\mathrm{C}_{20} \mathrm{H}_{26} \mathrm{NO}_{4}[\mathrm{M}+\mathrm{H}]^{+}:$344.1856; found 344.1860.

\section{Preparation of $( \pm)$-thebainone A (1)}

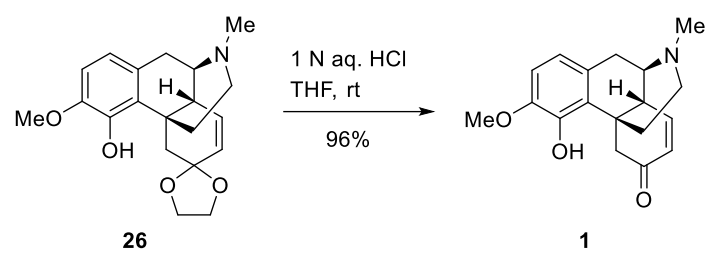

A solution of acetal $26(59.0 \mathrm{mg}, 0.172 \mathrm{mmol})$ in tetrahydrofuran $(3.4 \mathrm{~mL})$ was treated with $1 \mathrm{~N} \mathrm{HCl}(3.4 \mathrm{~mL})$. The mixture was stirred at room temperature for $3.5 \mathrm{~h}$, cooled to $0{ }^{\circ} \mathrm{C}$, and neutralized with concentrated ammonium hydroxide. The aqueous layer was extracted with $\mathrm{CH}_{2} \mathrm{Cl}_{2}(3 \times 3 \mathrm{~mL})$, and the combined organic layers were washed with saturated aqueous $\mathrm{NaCl}$ solution $(3 \mathrm{~mL})$ and dried over $\mathrm{MgSO}_{4}$. After removal of the solvent under vacuum, flash chromatography over silica gel $\left(\mathrm{CH}_{2} \mathrm{Cl}_{2} / \mathrm{MeOH}, 9: 1\right)$ yielded ( \pm )-thebainone A (1, $49.2 \mathrm{mg}$, $96 \%$ ) as a slightly yellow foam. 
1: $\mathrm{R}_{f}=0.28\left(\mathrm{CHCl}_{3} / \mathrm{MeOH}, 4: 1\right) .{ }^{1} \mathrm{H} \mathrm{NMR}\left(600 \mathrm{MHz}, \mathrm{CDCl}_{3}\right): \delta=1.89(\mathrm{td}, J=12.4,4.1 \mathrm{~Hz}$, 1 H, H-15), 1.93-1.97 (m, 1 H, H-15), 2.08 (td, $J=12.1,3.6$ Hz, 1 H, H-16a), 2.38 (d, $J=15.4$ Hz, 1 H, H-5b), 2.43 (s, 3 H, NMe) 2.53-2.58 (m, 1 H, H-16b), 2.66 (dd, J = 18.3, 5.5 Hz, 1 H, H-10a) 2.92 (br s, $1 \mathrm{H}, \mathrm{H}-14$ ), 3.01 (d, $J=18.3 \mathrm{~Hz}, 1 \mathrm{H}, \mathrm{H}-10 \mathrm{~b}), 3.24$ (t, $J=4.1 \mathrm{~Hz}, 1 \mathrm{H}, \mathrm{H}-9$ ), $3.80(\mathrm{~s}, 3 \mathrm{H}, \mathrm{OMe}), 4.26$ (d, $J=15.8 \mathrm{~Hz}, 1 \mathrm{H}, \mathrm{H}-5 \mathrm{a}), 5.88$ (ddd, $J=9.8,3.0,1.1 \mathrm{~Hz}, 1 \mathrm{H}, \mathrm{H}-7)$, 6.11 (br s, $1 \mathrm{H}$, phenol), 6.54 (d, $J=8.3 \mathrm{~Hz}, 1 \mathrm{H}, \mathrm{H}-1$ ), 6.64 (d, $J=8.3 \mathrm{~Hz}, 1 \mathrm{H}, \mathrm{H}-2) 6.67$ (dd, $J=9.8,1.9 \mathrm{~Hz}, 1 \mathrm{H}, \mathrm{H}-8) \mathrm{ppm} .{ }^{13} \mathrm{C} \mathrm{NMR}\left(151 \mathrm{MHz}, \mathrm{CDCl}_{3}\right): \delta=24.4$ (t, C-10), 36.3 (t, C-15), 40.5 (s, C-13), 42.6 (q, NMe), 47.0 (d, C-14), 47.2 (t, C-16), 49.0 (t, C-5), 56.0 (q, OMe), 56.2 (d, C-9), 108.8 (d, C-2), 118.3 (d, C-1), 122.8 (s, C-12), 130.2 (s, C-11) 131.0 (d, C-7), 144.6 (s, C-4), 144.9 (s, C-3), 149.4 (d, C-8) 199.4 (s, C-6) ppm. IR (ATR): v = 3019 (w), 2926 (w), 2840 (w), 2808 (w), 1676 (s), 1655 (m), 1483 (s), 1438 (m), 1275 (s), 1258 (s), 1230 (s), 1149 (m), 1101 (m), 1052 (s), 1023 (m), 989 (m), 855 (m), 793 (m), 740 (s) cm . ESI-MS: m/z = $300.2[\mathrm{M}+\mathrm{H}]^{+}, 599.3[2 \mathrm{M}+\mathrm{H}]^{+}$. HRMS (ESI): m/z calcd for $\mathrm{C}_{18} \mathrm{H}_{22} \mathrm{NO}_{3}[\mathrm{M}+\mathrm{H}]^{+}: 300.1594$; found 300.1594 .

Table S1. Comparison of ${ }^{13} \mathrm{C}$ NMR Data [ppm] for 1 with Literature ${ }^{5,6}$ Data.

\begin{tabular}{llllll}
\hline This Work & Ref 5 & This Work & Ref $5^{\mathrm{b}}$ & This Work $^{\mathrm{a}}$ & Ref 5 $^{\mathrm{b}}$ \\
\hline 24.4 & 24.4 & 49.0 & 48.9 & 130.2 & 130.2 \\
36.3 & 36.2 & 56.0 & 55.9 & 131.0 & 130.8 \\
40.5 & 40.5 & 56.2 & 56.1 & 144.6 & 144.6 \\
42.6 & 42.5 & 108.8 & 108.8 & 144.9 & 144.9 \\
47.0 & 47.0 & 118.3 & 118.2 & 149.4 & 149.4 \\
47.2 & 47.1 & 122.8 & 122.8 & 199.4 & 199.3 \\
\hline
\end{tabular}

a $151 \mathrm{MHz}, \mathrm{CDCl}_{3},{ }^{\mathrm{b}} 50 \mathrm{MHz}, \mathrm{CDCl}_{3}$.

\section{References}

(1) Markovich, K. M.; Tantishaiyakul, V.; Hamada, A.; Miller, D. D.; Romstedt, K. J.; Shams, G.; Shin, Y.; Fraundorfer, P. F.; Doyle, K.; Feller, D. R. J. Med. Chem. 1992, 35, 466-479.

(2) a) Koizumi, H.; Yokoshima, S.; Fukuyama, T. Chem. Asian J. 2010, 5, 2192-2198; b) Uchida, K.; Yokoshima, S.; Kan, T.; Fukuyama, T. Heterocycles 2009, 77, 1219-1234; c) Uchida, K.; Yokoshima, S.; Kan, T.; Fukuyama, T. Org. Lett. 2006, 8, 5311-5313.

(3) a) Varin, M.; Barré, E.; Iorga, B.; Guillou, C. Chem. Eur. J. 2008, 14, 6606-6608; b) Guillou, C.; Beunard, J.-L.; Gras, E.; Thal, C. Angew. Chem. 2001, 113, 4881-4882; Angew. 
Chem. Int. Ed. 2001, 40, 4745-4746; c) Still, W. C.; Lewis, A. J.; Goldsmith, D. Tetrahedron Lett. 1971, 12, 1421-1424.

(4) Guibe-Jampel, E.; Wakselman, M. J. Chem. Soc. Chem. Commun. 1980, 993-994.

(5) Szabó, Z.; Marton, J.; Hosztafi, S. Magn. Reson. Chem. 1995, 33, 913-916.

(6) See also: a) Zhang, A.; Li, F.; Ding, C.; Yao, Q.; Knapp, B. I.; Bidlack, J. M.; Neumeyer, J. L. J. Med. Chem. 2007, 50, 2747-2751; b) Davidson, M. R.; Gregg, B. T. Synth. Commun. 1998, 28, 547-558. 


\section{NMR spectra}

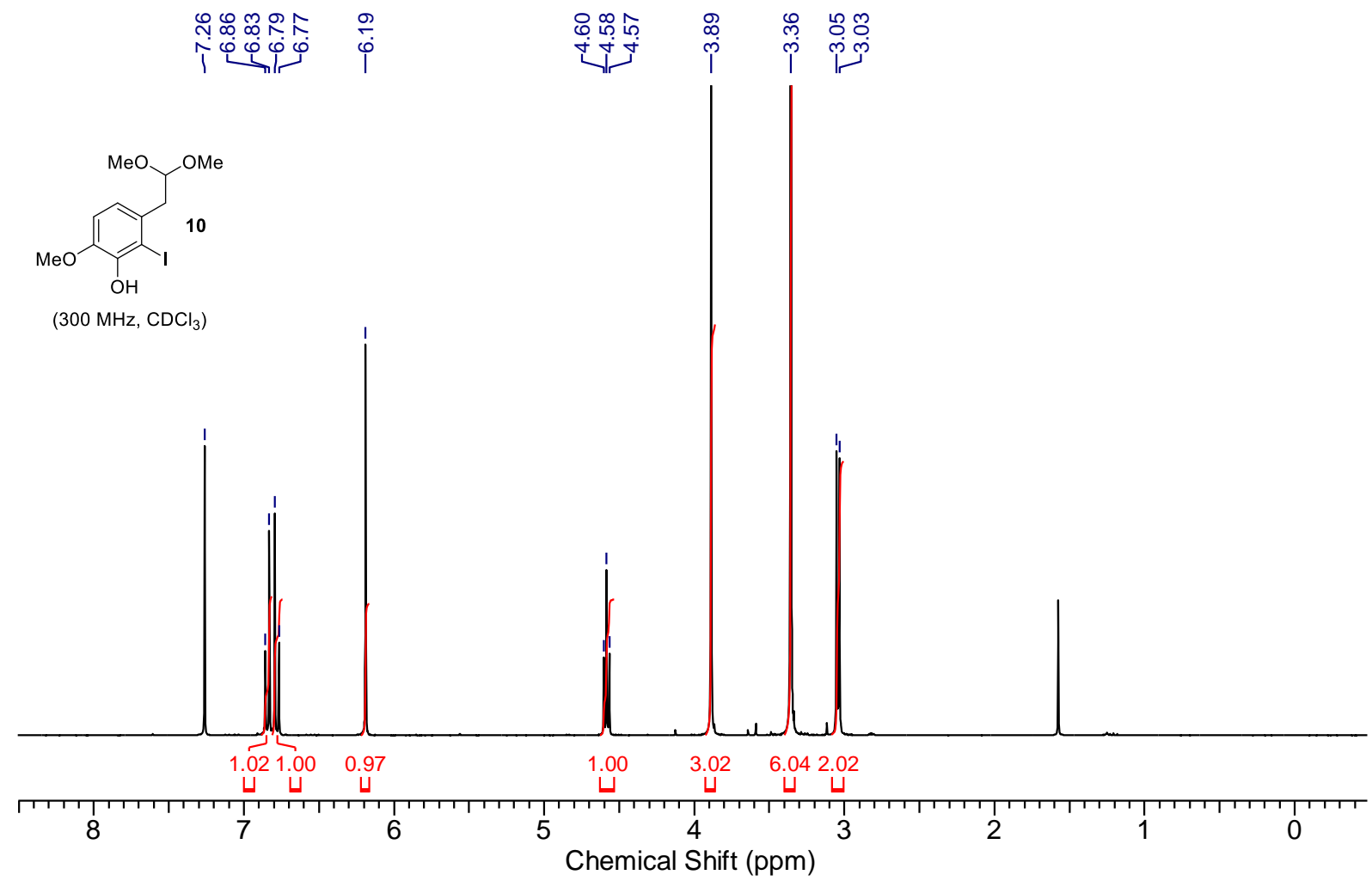




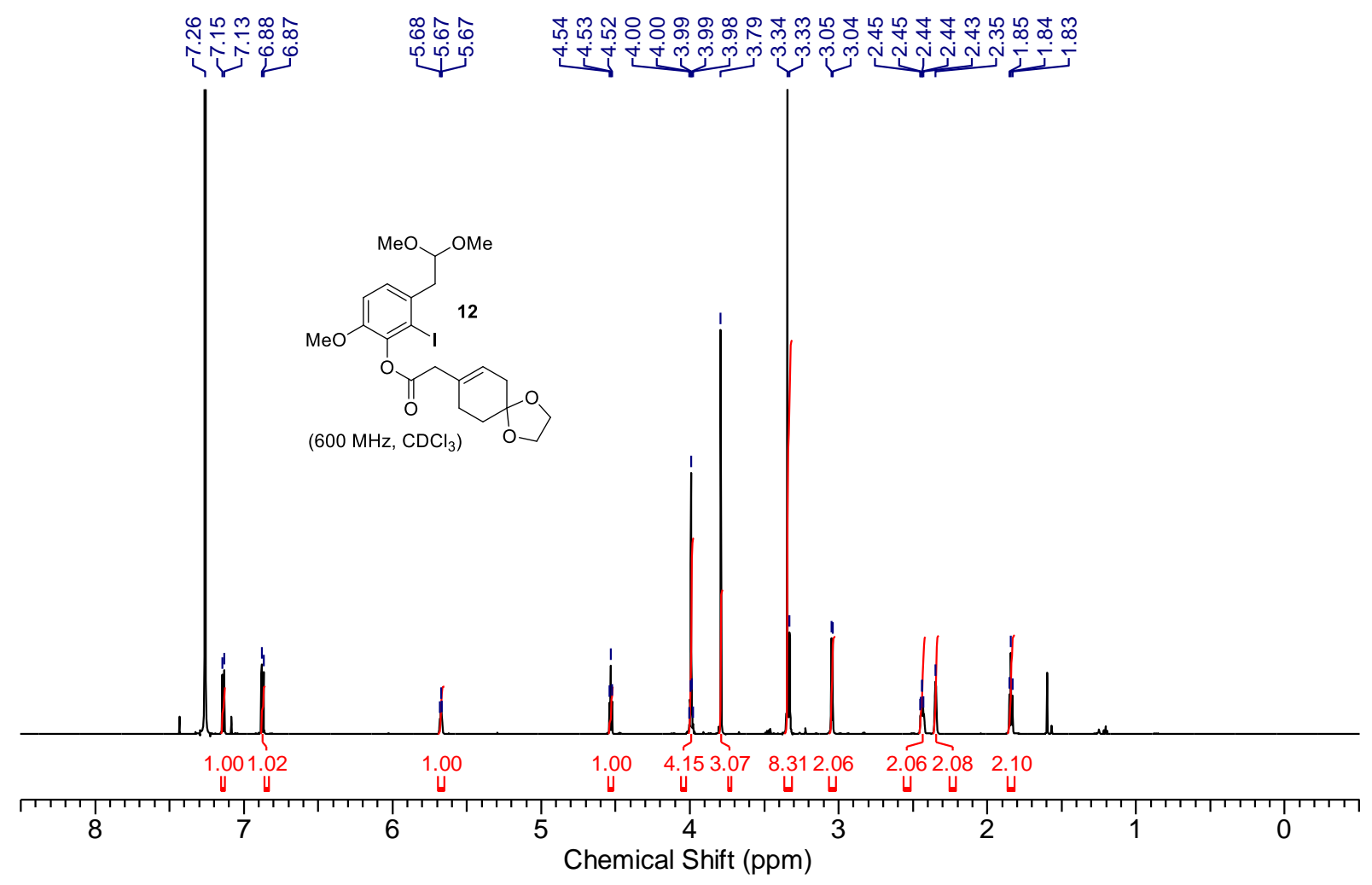

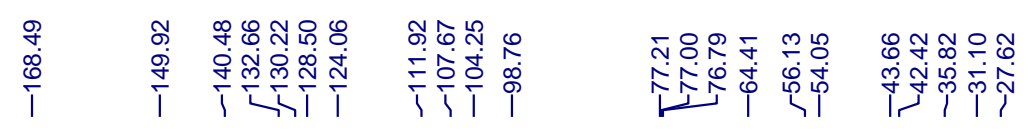

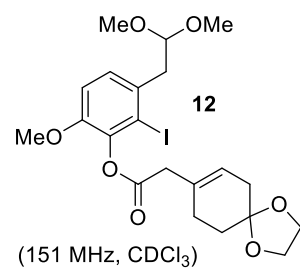

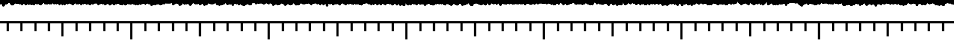

200

180

160

140

120

100

80

60

60

40

20

Chemical Shift (ppm) 

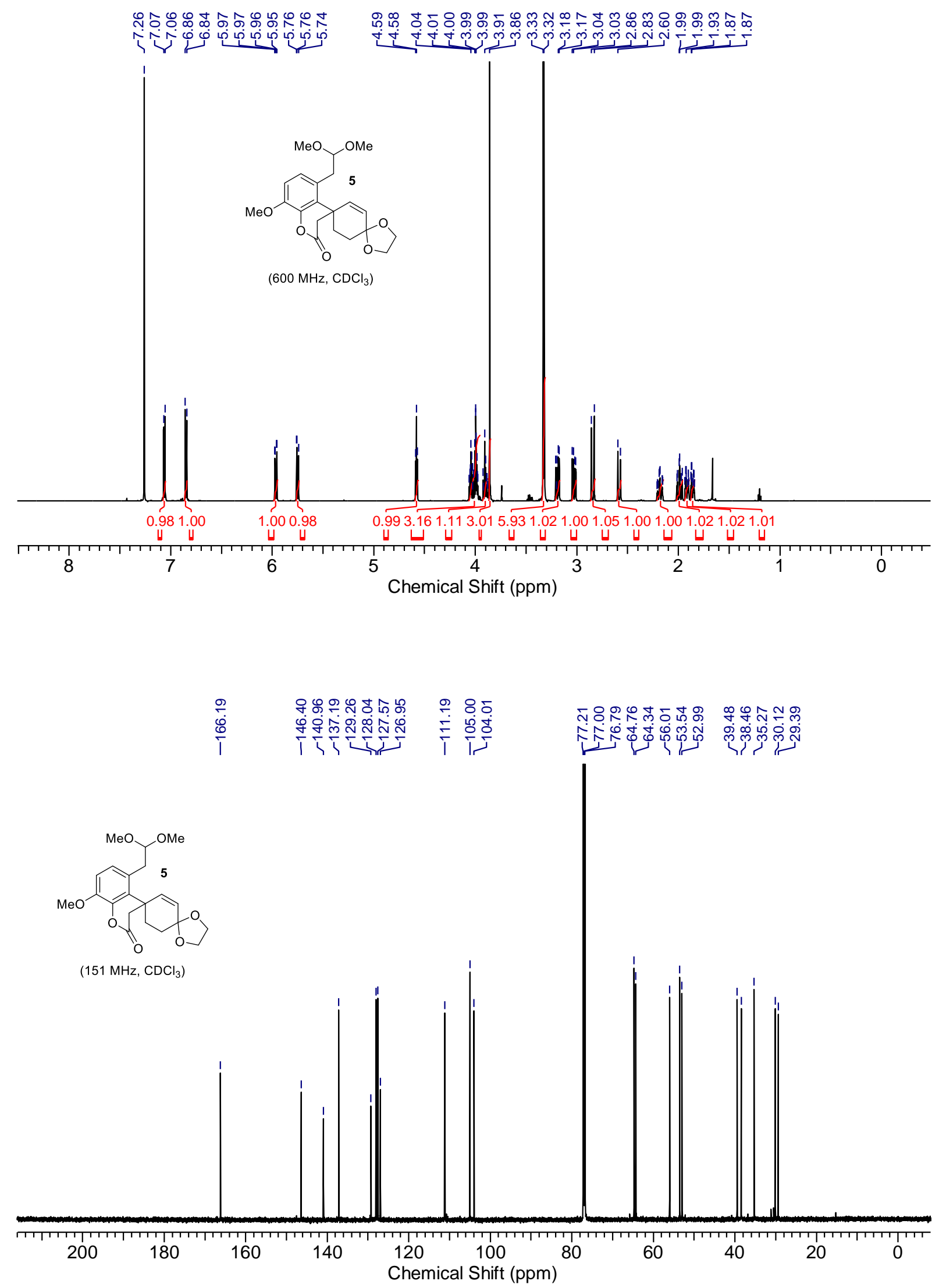

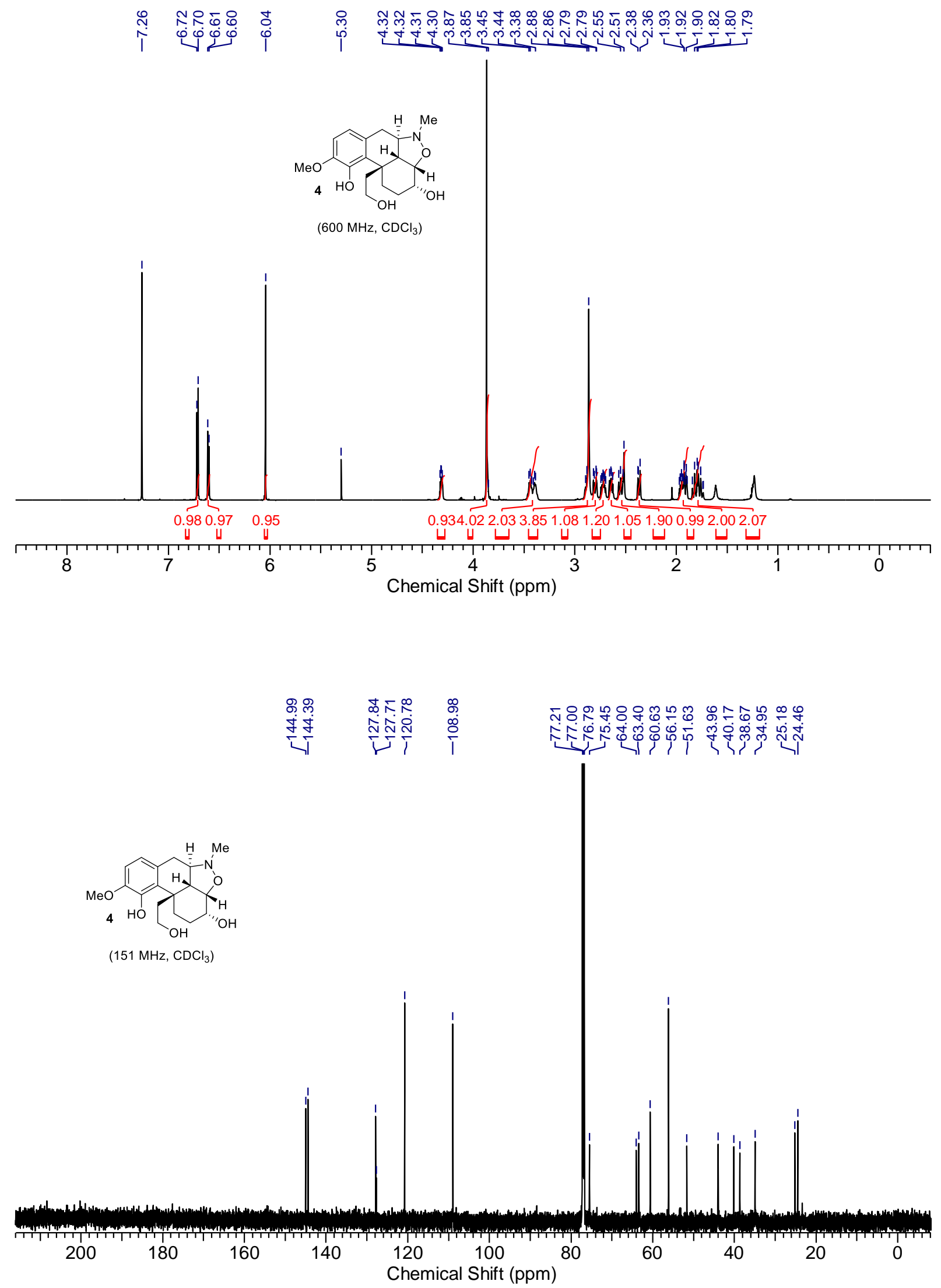

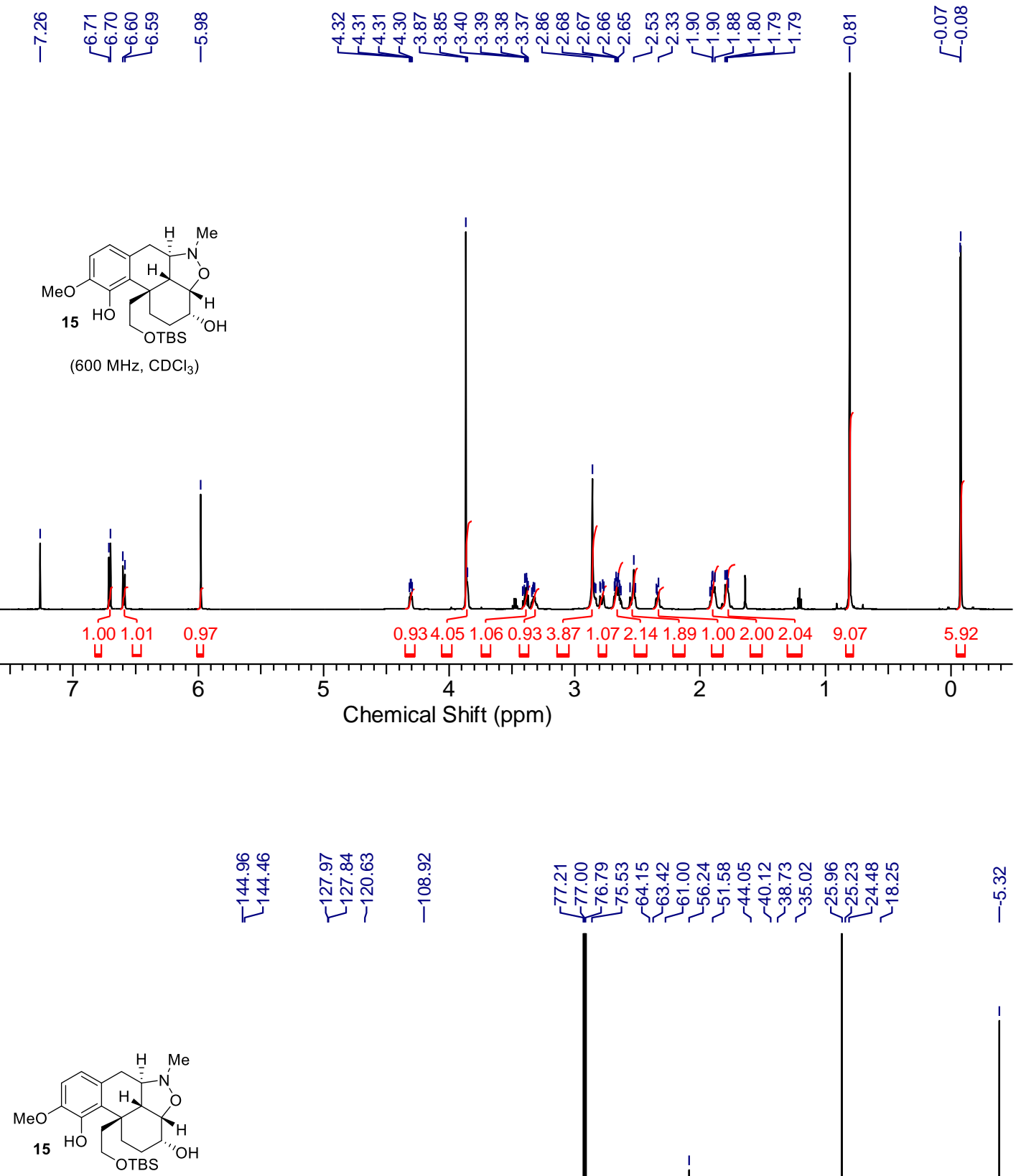

(151 MHz, $\mathrm{CDCl}_{3}$ )

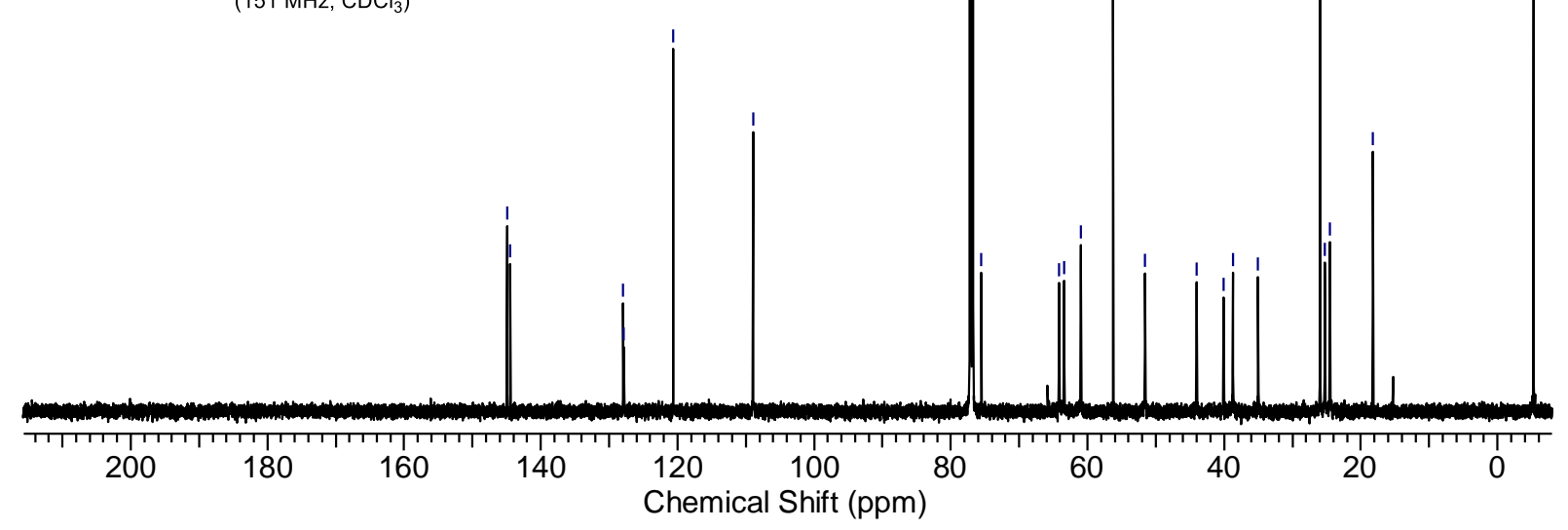



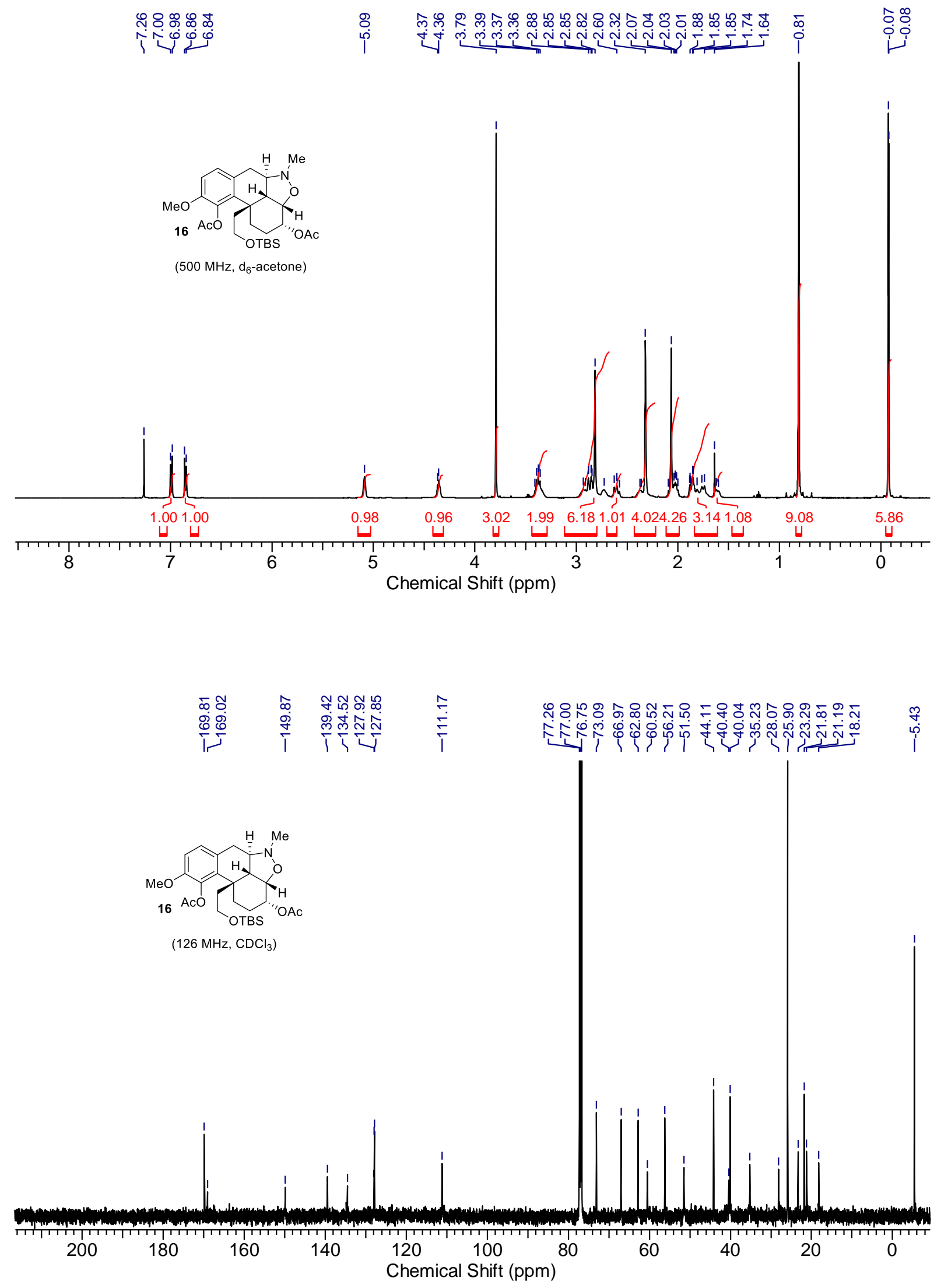

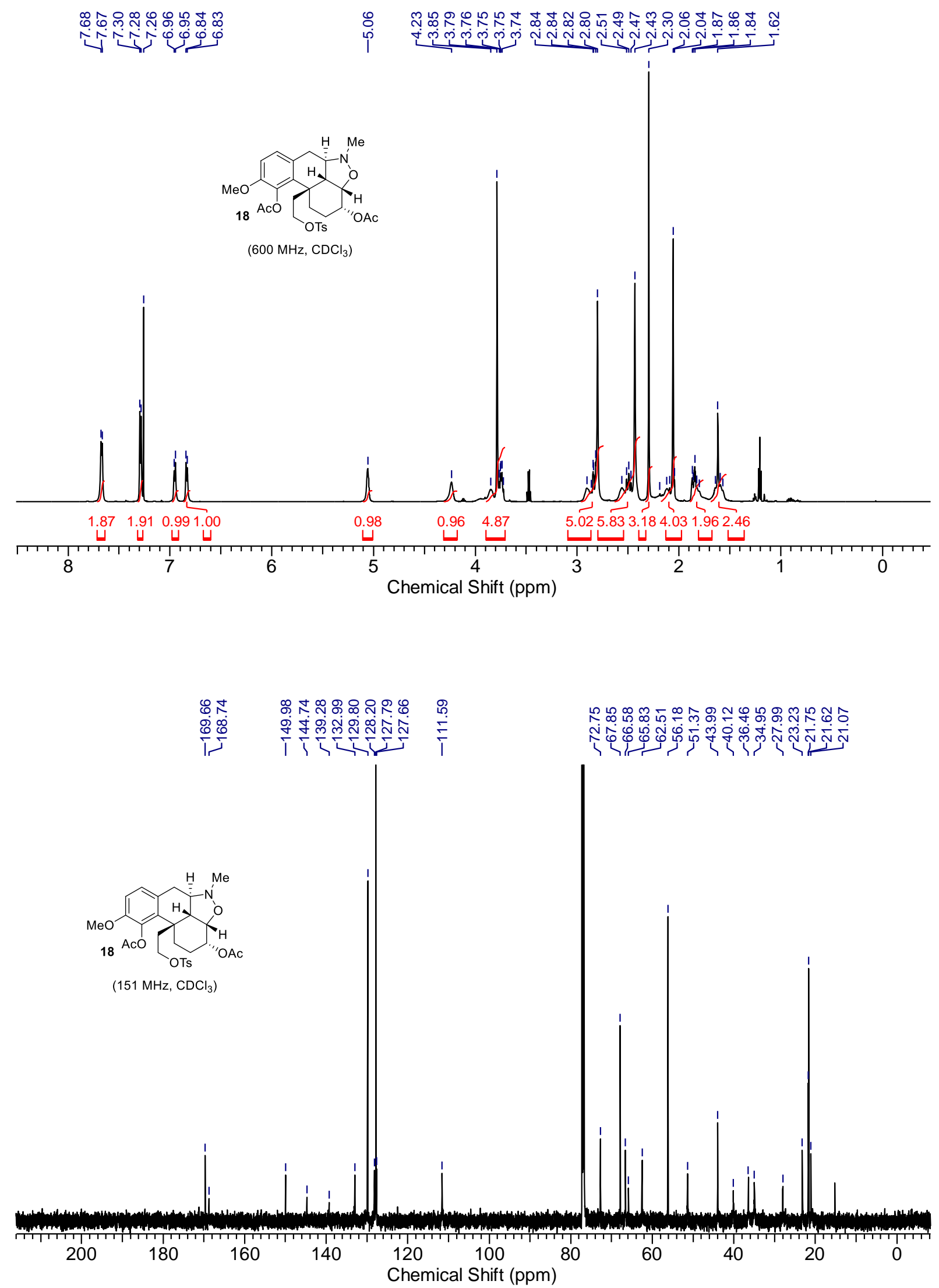


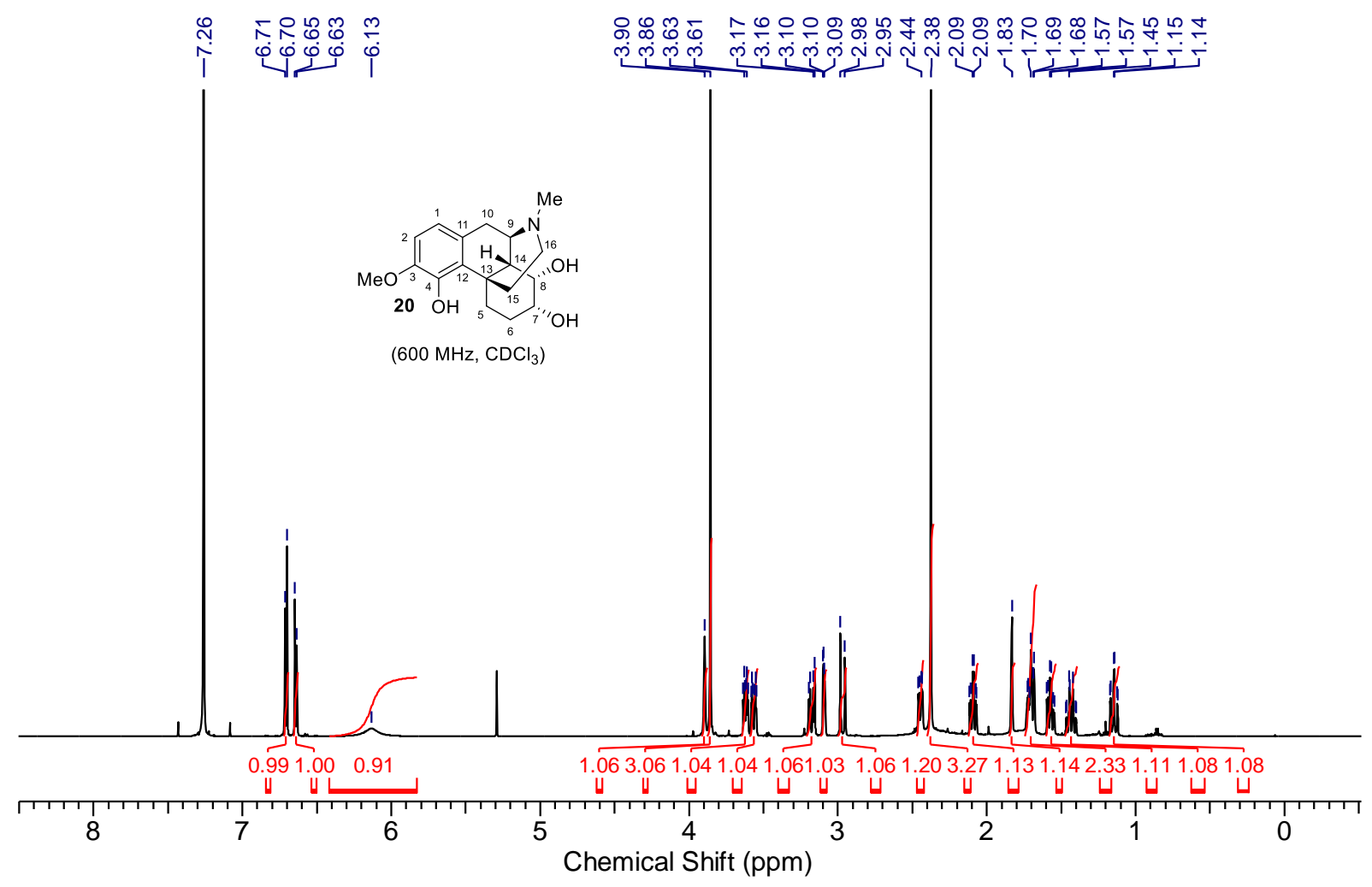

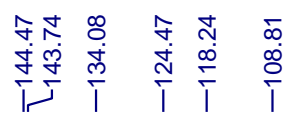

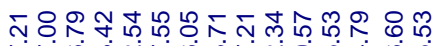

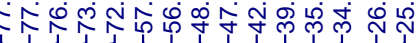

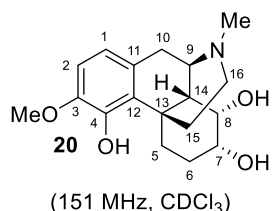

(151 MHz, $\mathrm{CDCl}_{3}$ )

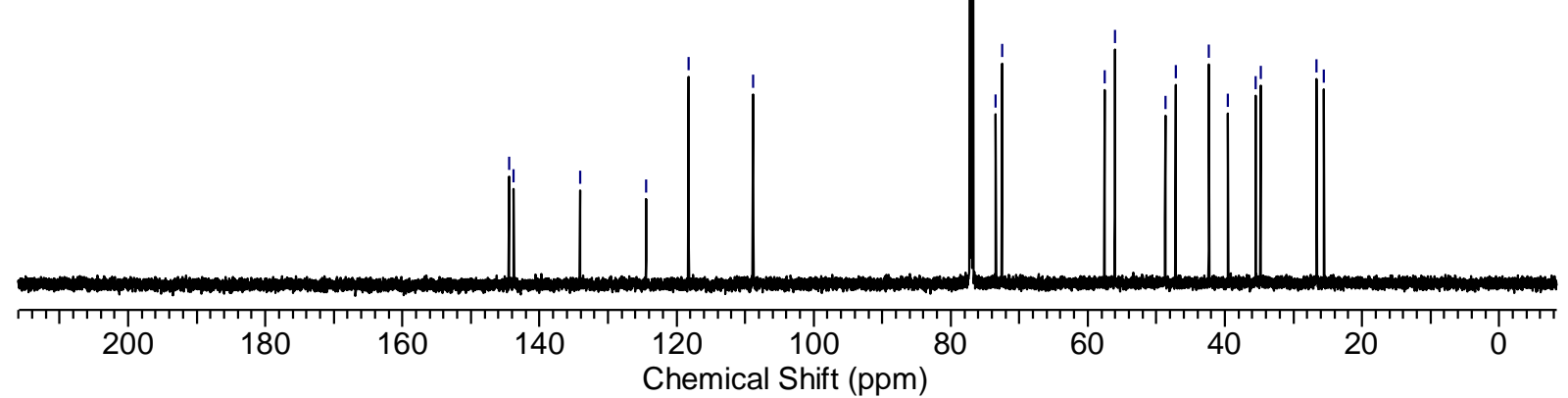



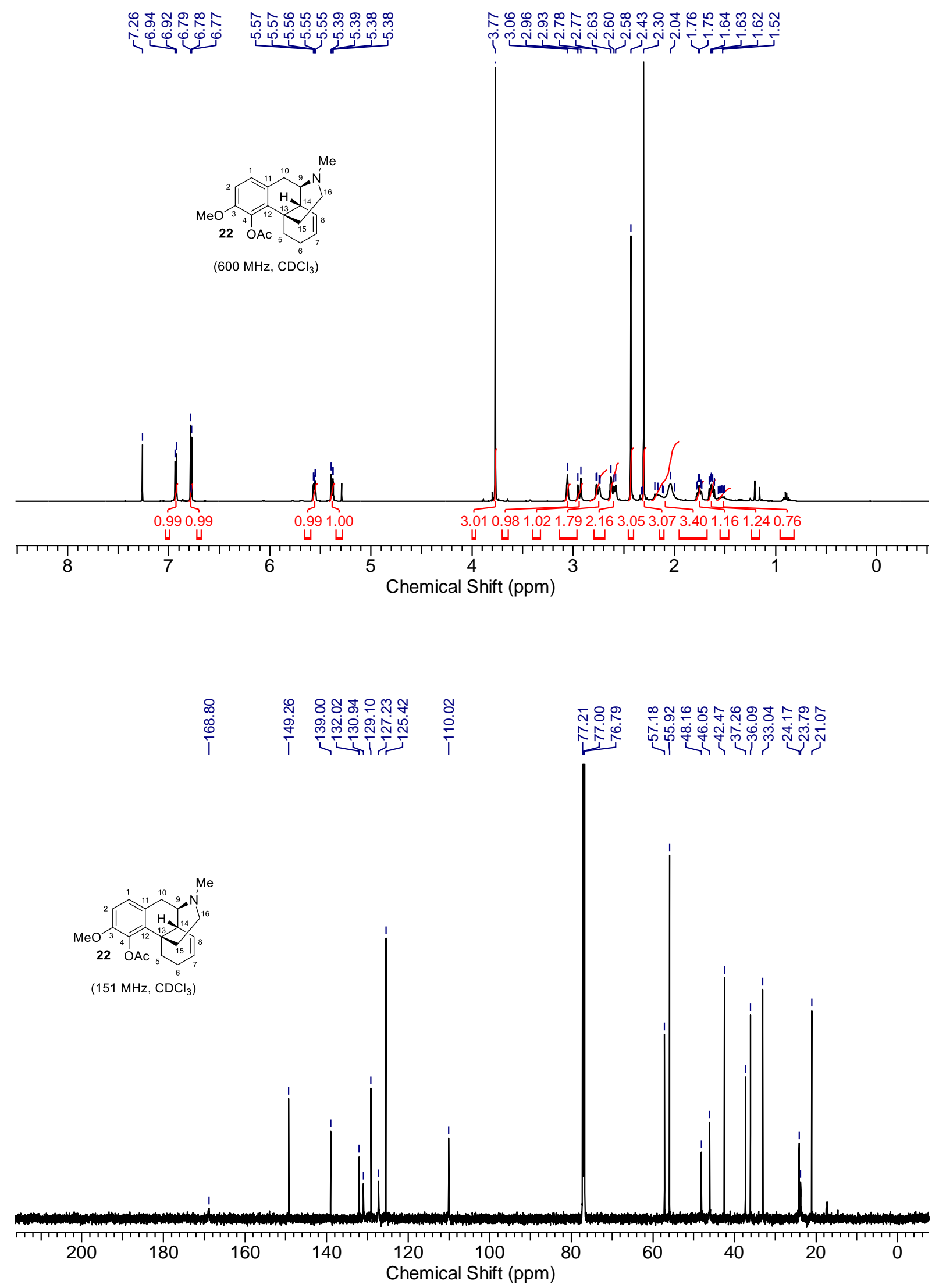

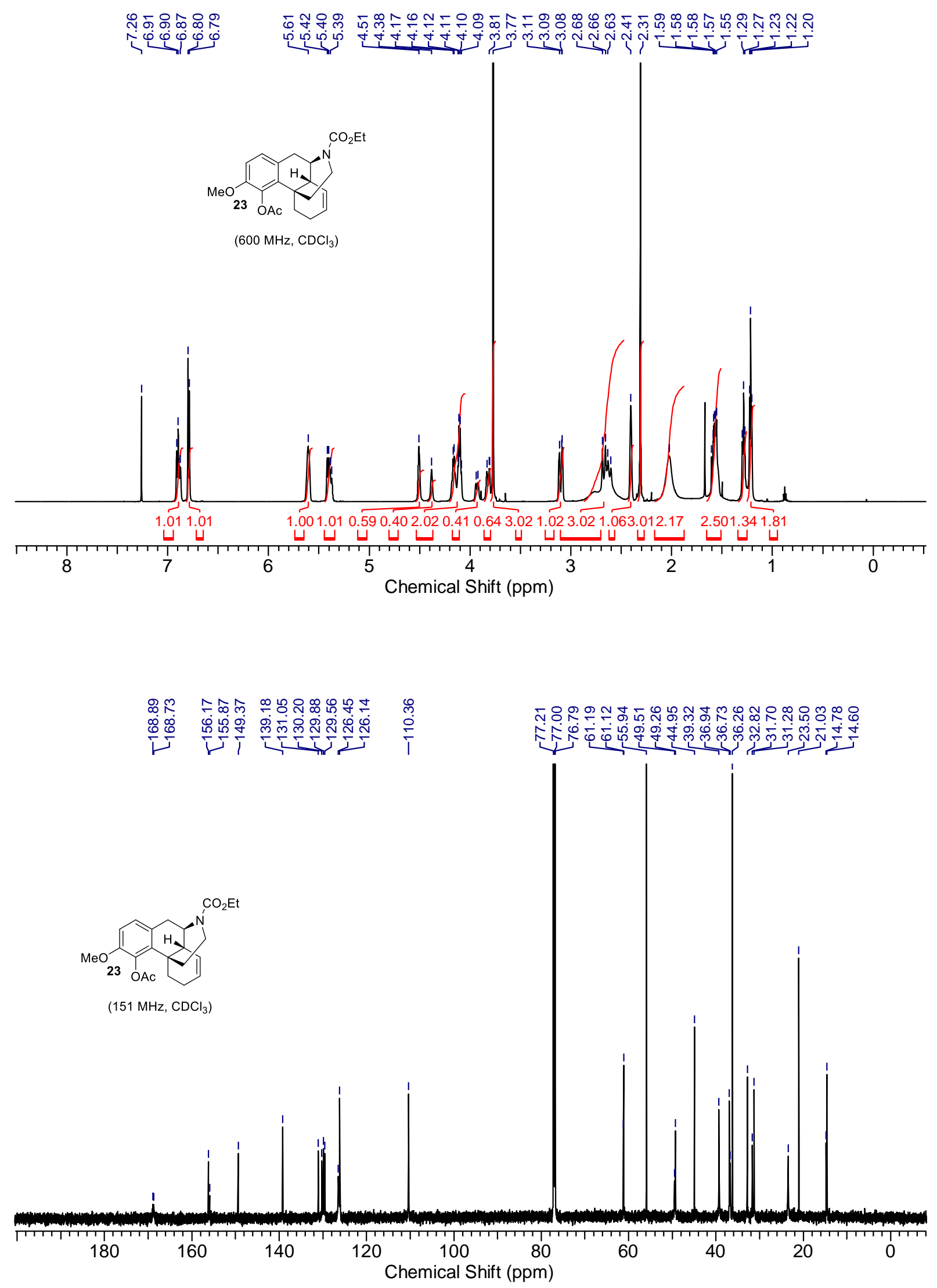


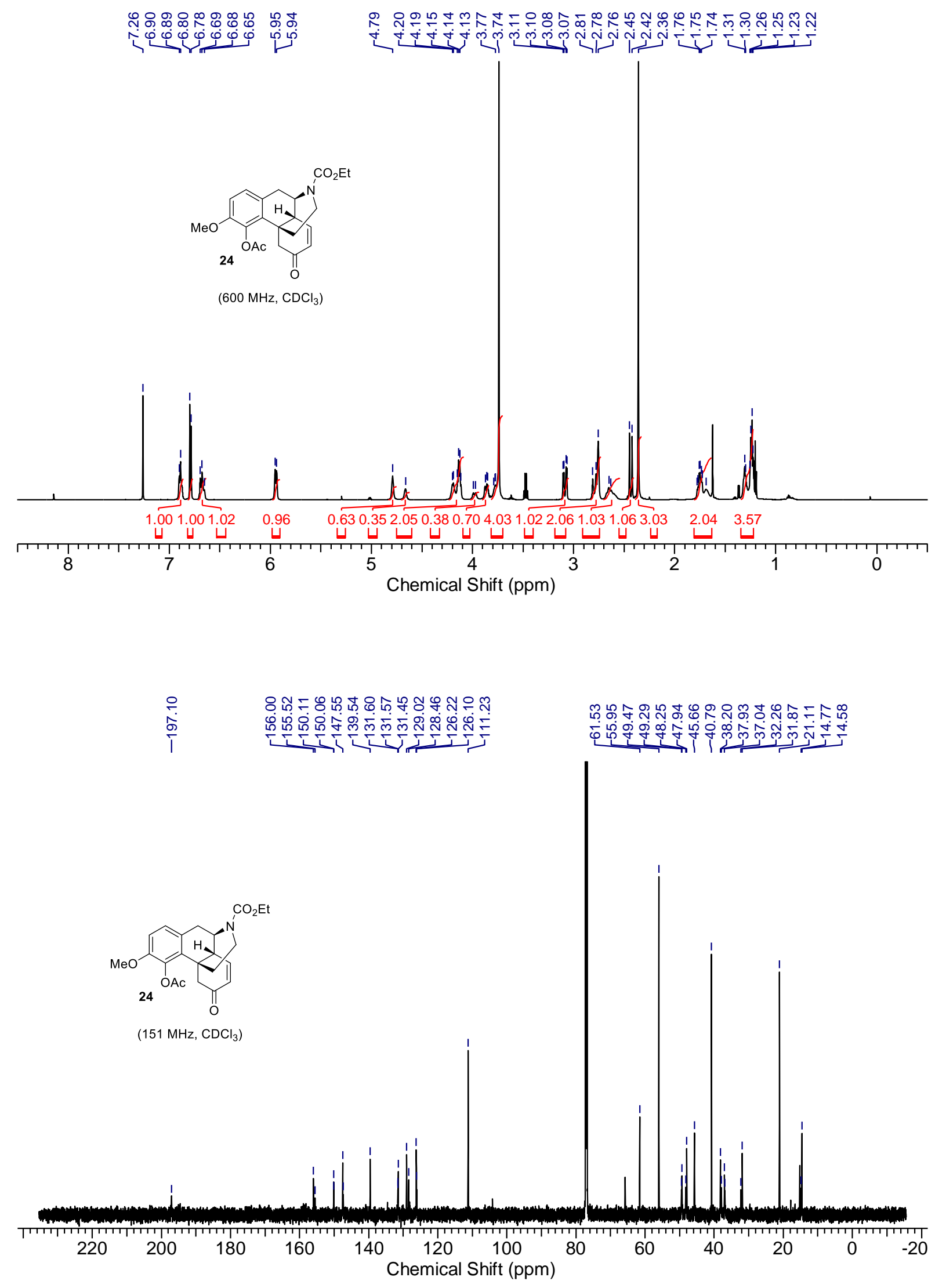



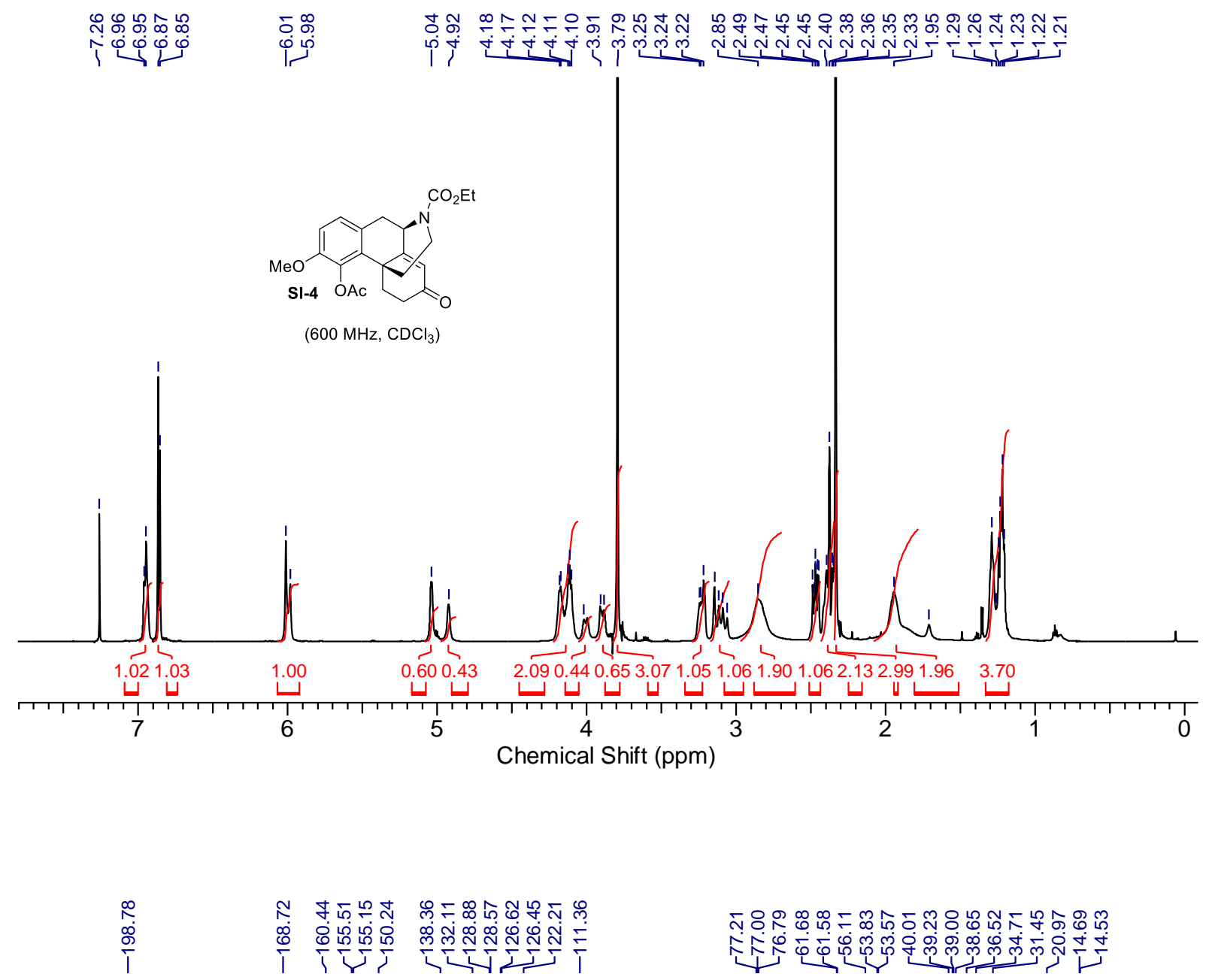

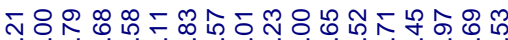

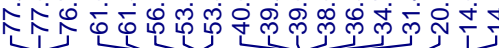

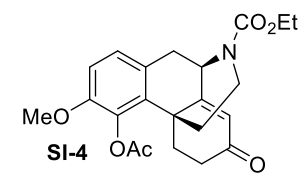

(151 MHz, $\mathrm{CDCl}_{3}$ )

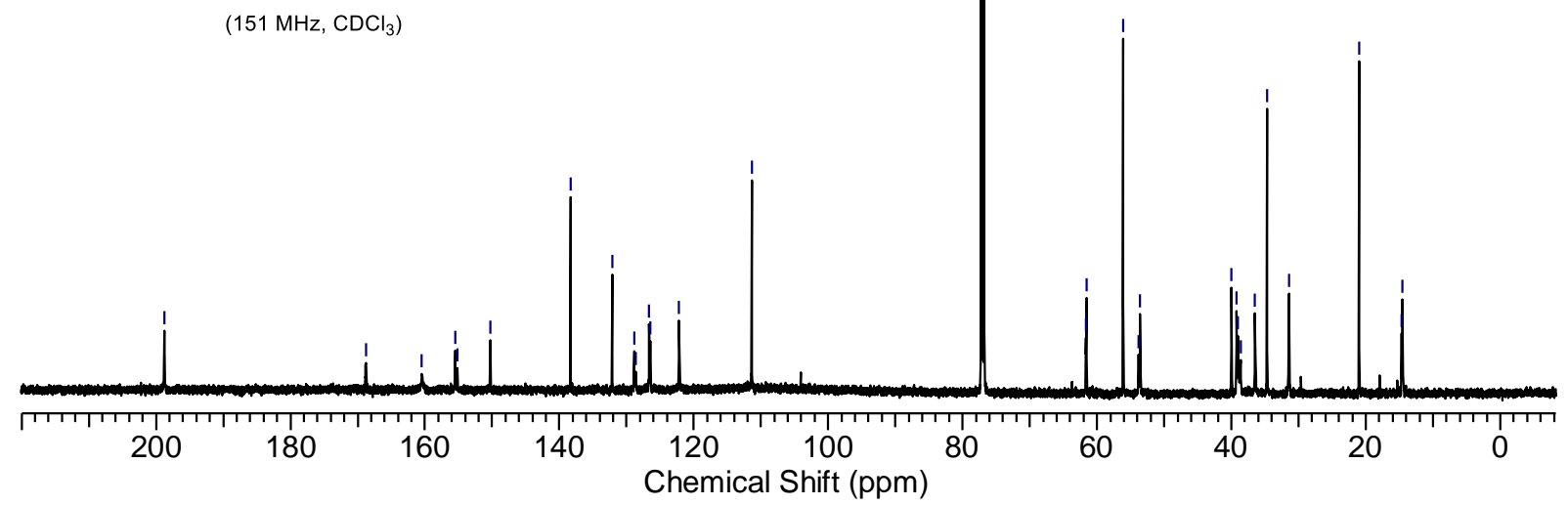




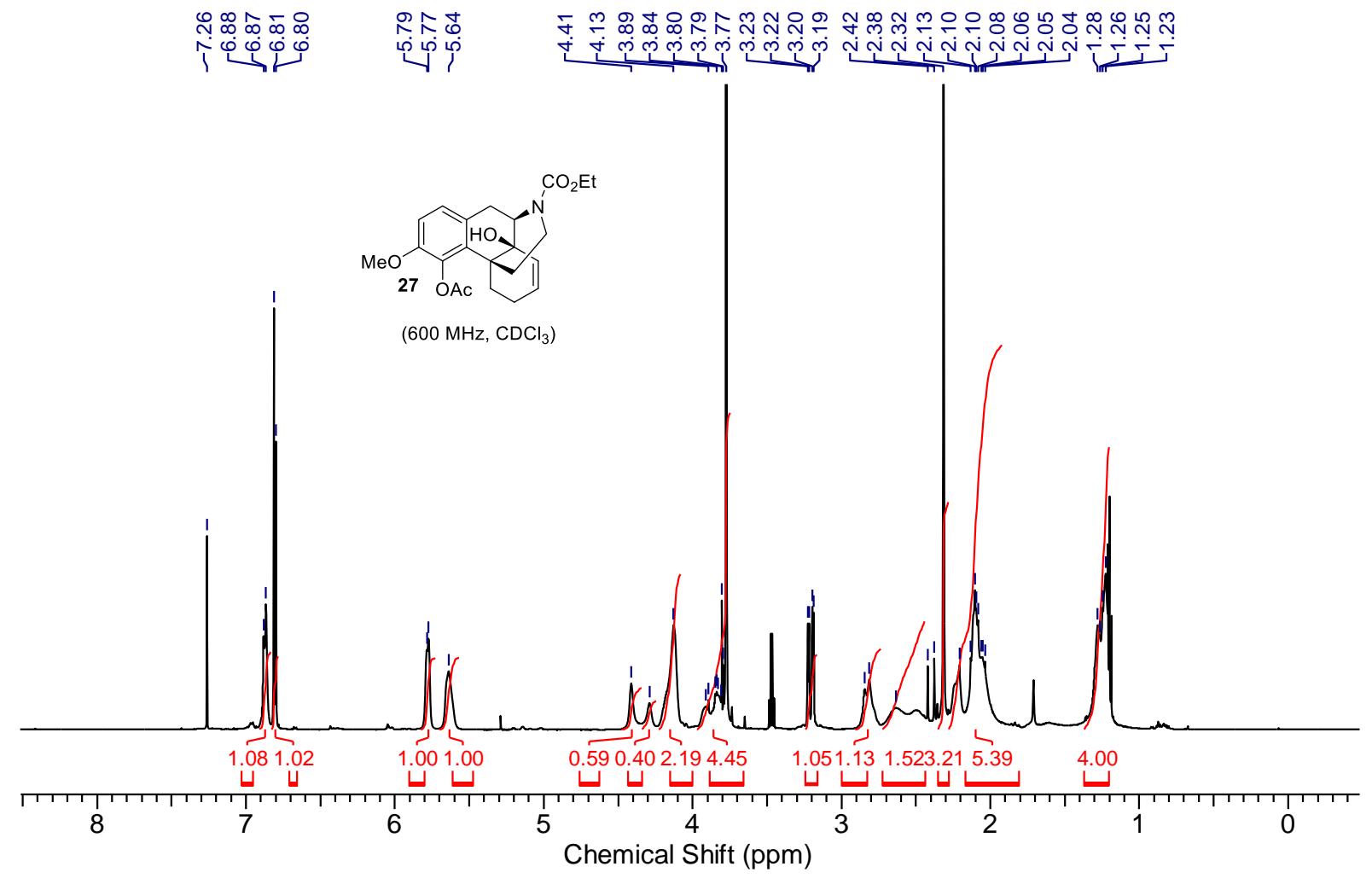



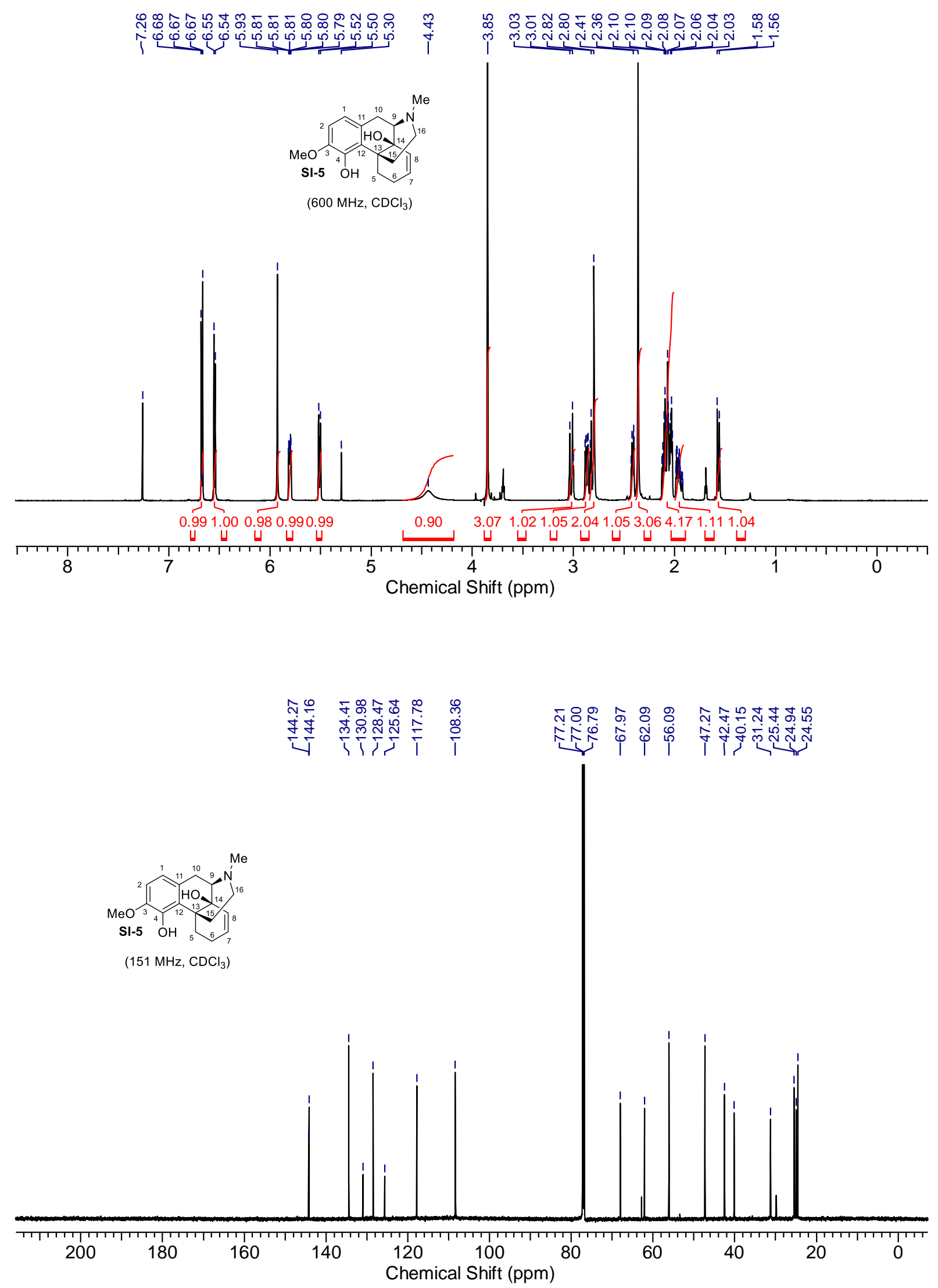

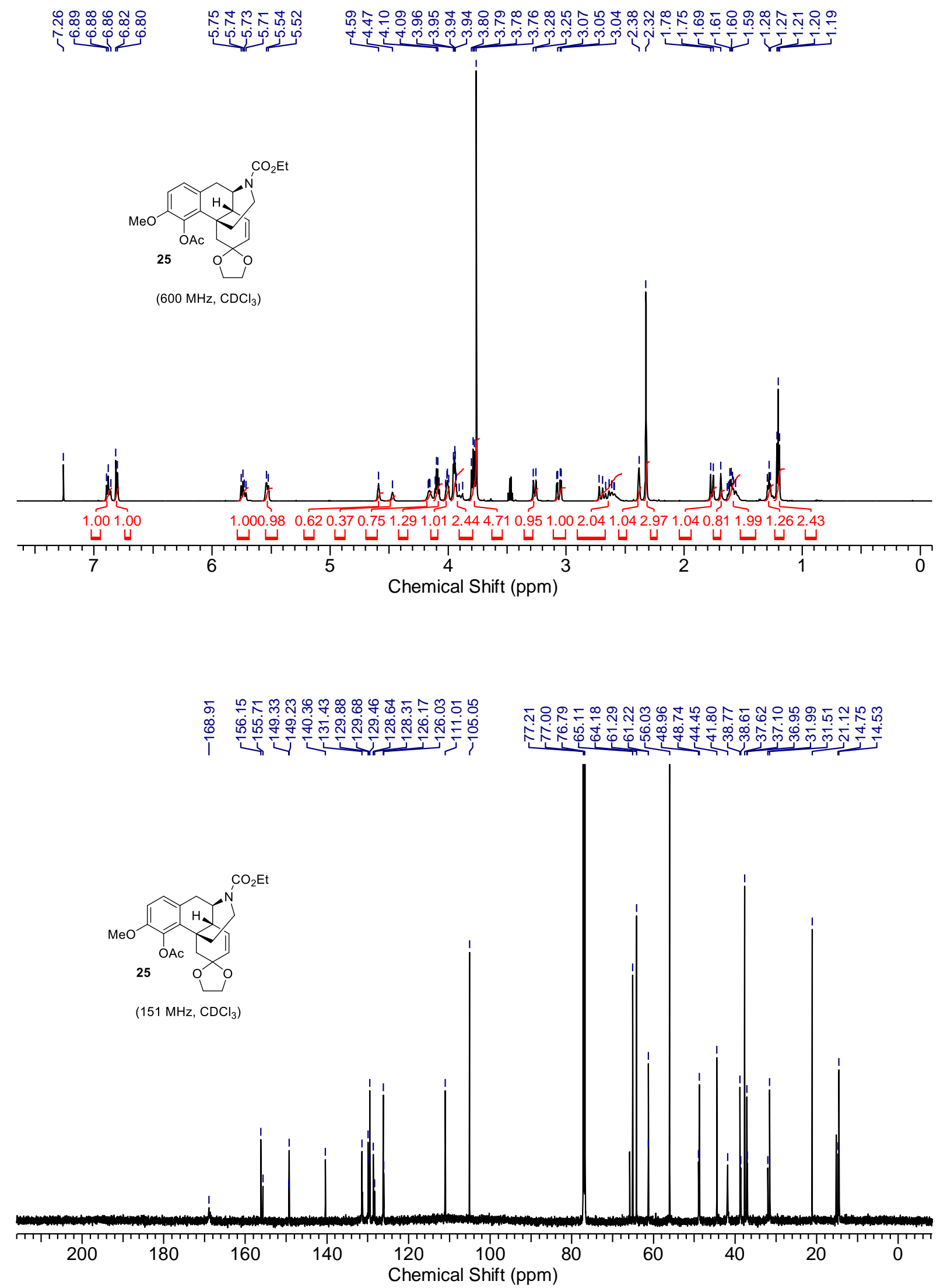


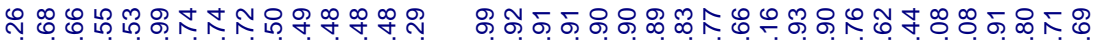

个

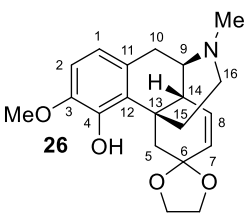

$\left(600 \mathrm{MHz}, \mathrm{CDCl}_{3}\right)$
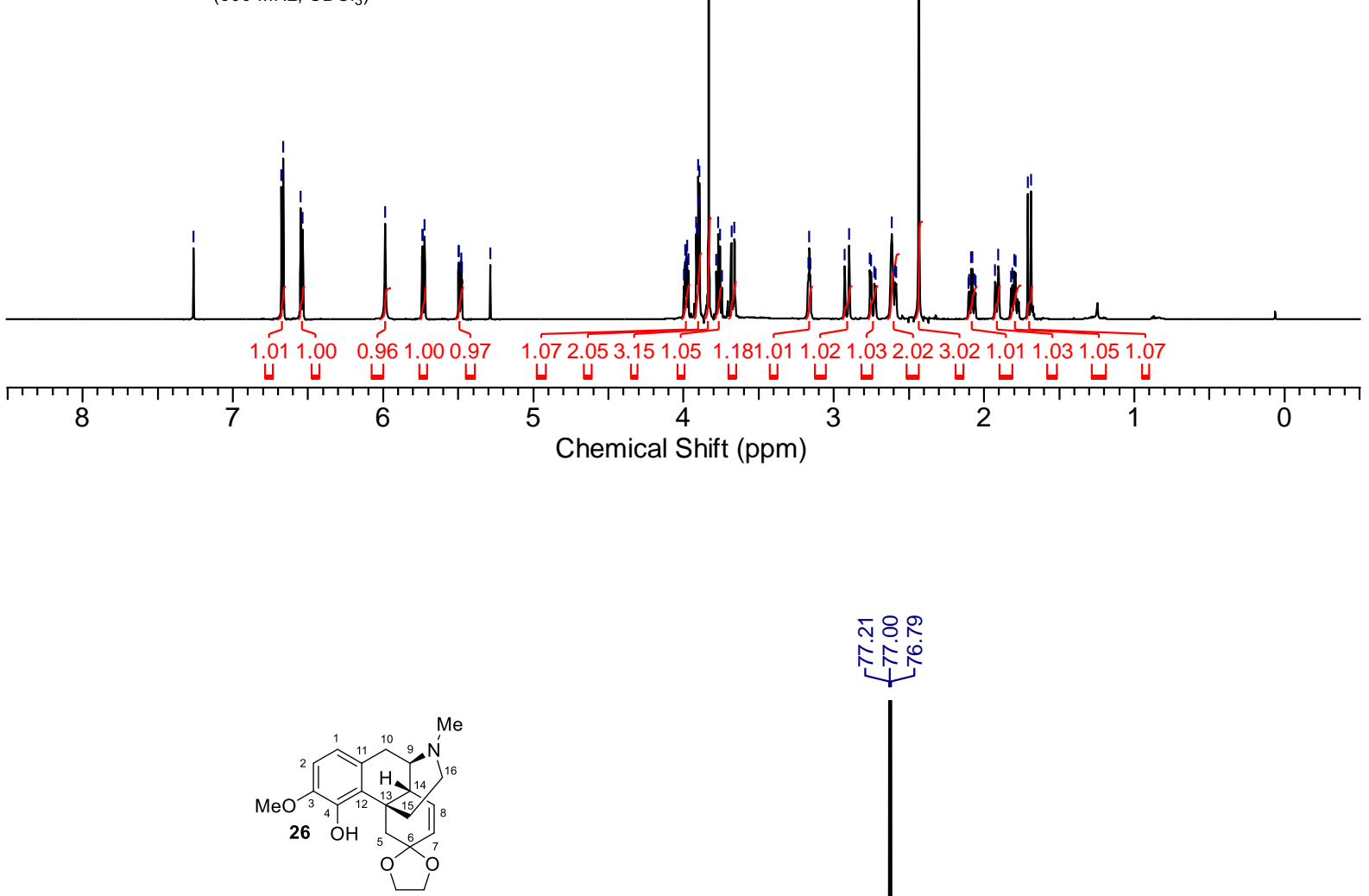

$\sin 8$

余余

$\left(151 \mathrm{MHz}, \mathrm{CDCl}_{3}\right)$

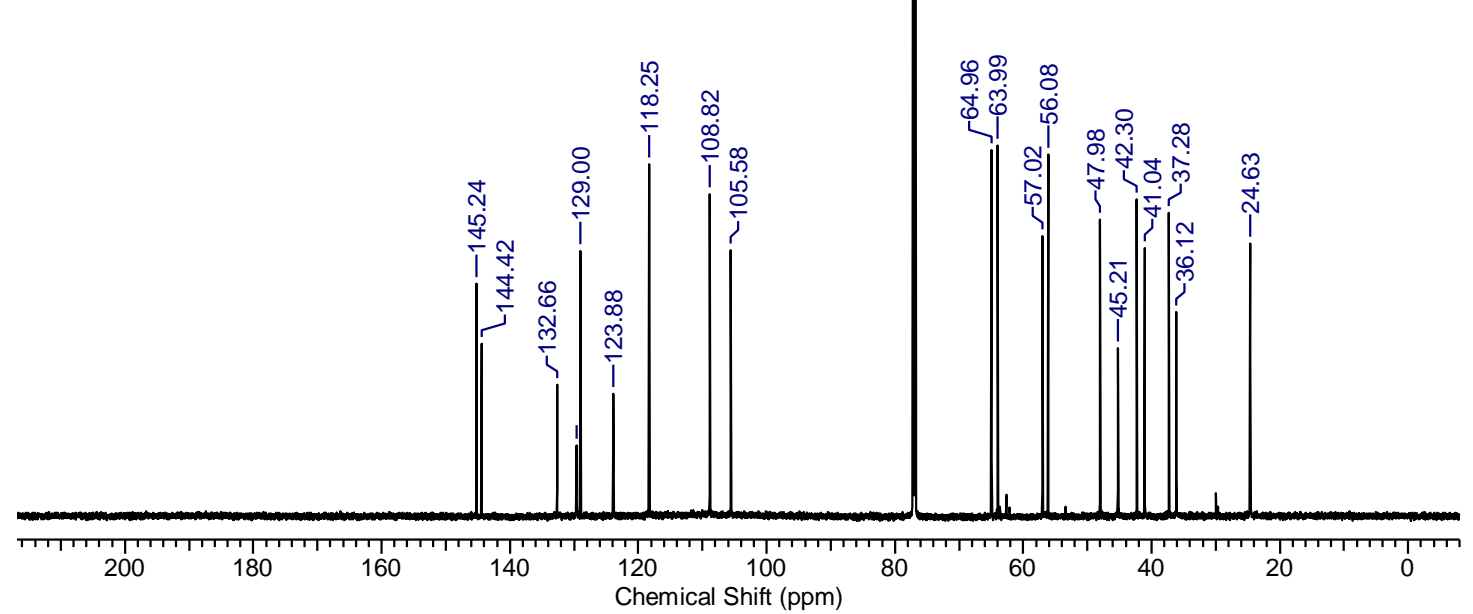




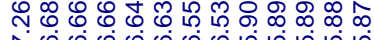

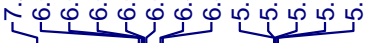

(600 MHz, $\mathrm{CDCl}_{3}$ )

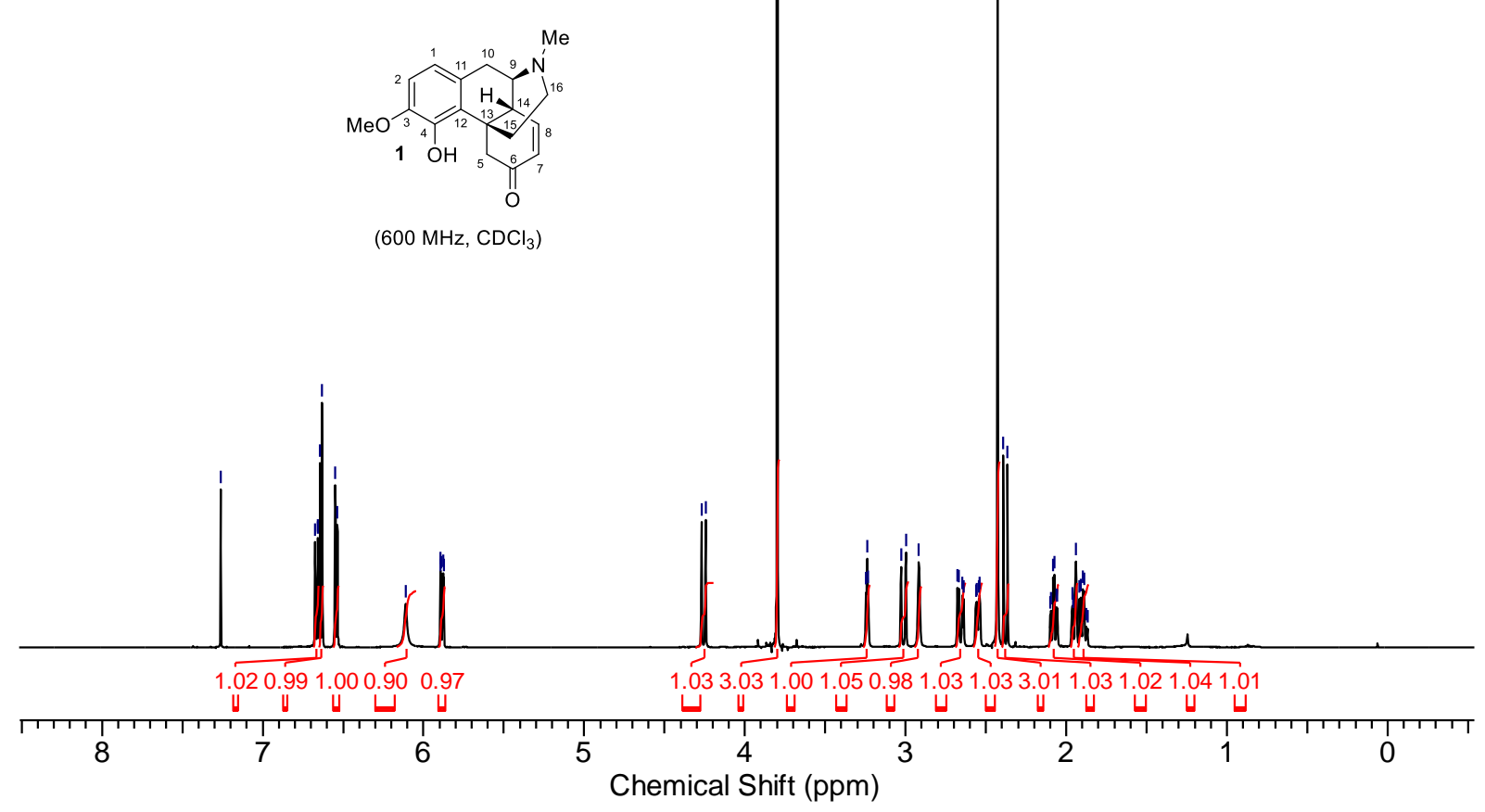

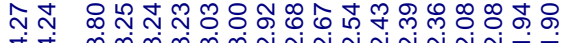

ن

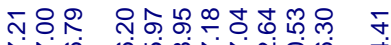

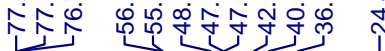

(1)

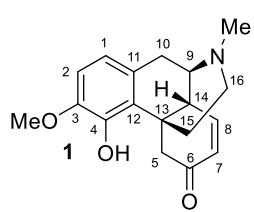

(151 MHz, $\mathrm{CDCl}_{3}$ )

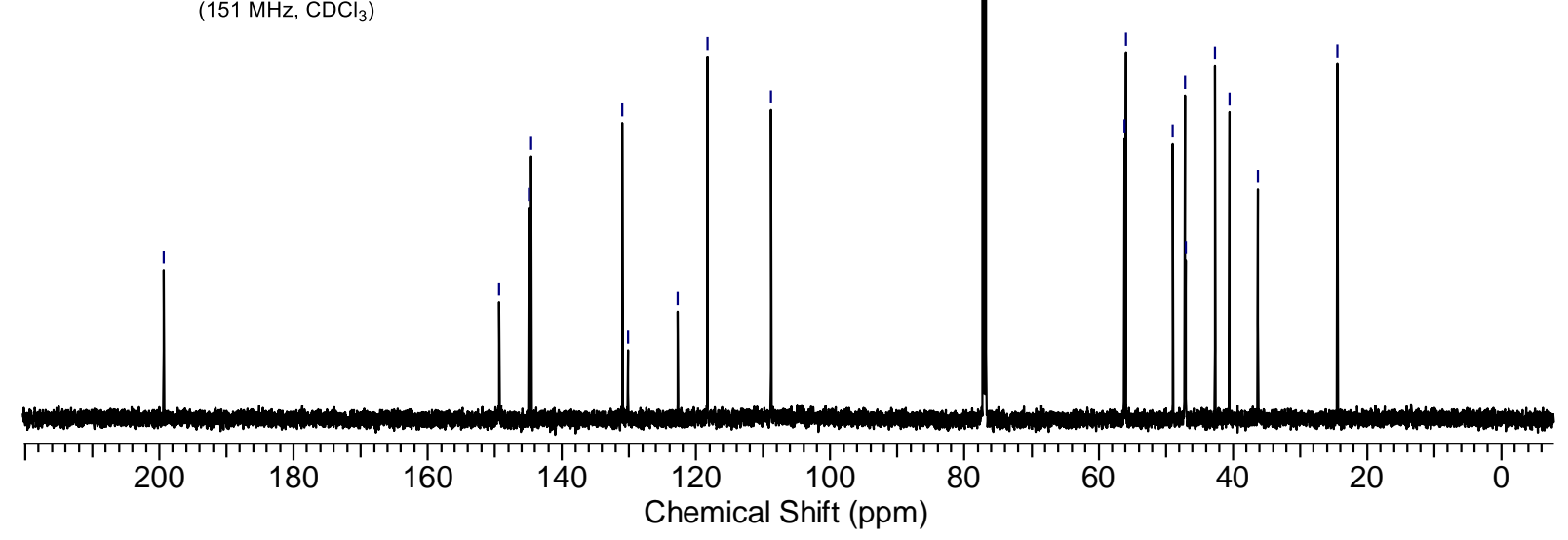

